\title{
THE
}

$1-19-2021$

\section{Computational Study of Helical and Helix-Hinge-Helix Conformations of an Anti-Microbial Peptide in Solution by Molecular Dynamics and Vibrational Analysis}

\author{
Faramarz Joodaki \\ University of Rhode Island \\ Lenore M. Martin \\ University of Rhode Island, martin@uri.edu \\ Michael L. Greenfield \\ University of Rhode Island, greenfield@uri.edu
}

Follow this and additional works at: https://digitalcommons.uri.edu/che_facpubs

Part of the Chemical Engineering Commons

This is a pre-publication author manuscript of the final, published article.

Terms of Use

All rights reserved under copyright.

\section{Citation/Publisher Attribution}

Joodaki, Faramarz, Lenore M. Martin and Michael L. Greenfield. Computational Study of Helical and HelixHinge-Helix Conformations of an Anti-Microbial Peptide in Solution by Molecular Dynamics and Vibrational Analysis. J. Phys. Chem. B, January 19, 2021. https://doi.org/10.1021/acs.jpcb.0c07988

This Article is brought to you for free and open access by the Chemical Engineering at DigitalCommons@URI. It has been accepted for inclusion in Chemical Engineering Faculty Publications by an authorized administrator of DigitalCommons@URI. For more information, please contact digitalcommons-group@uri.edu. 


\title{
Computational Study of Helical and
}

\section{Helix-Hinge-Helix Conformations of an}

Anti-Microbial Peptide in Solution by

\section{Molecular Dynamics and Vibrational Analysis}

\author{
Faramarz Joodaki ${ }^{\dagger}$ Lenore M. Martin ${ }^{\ddagger}{ }^{\ddagger}$ and Michael L. Greenfield ${ }^{*} \dagger$ \\ $\dagger$ †epartment of Chemical Engineering, University of Rhode Island, Kingston RI 02881 \\ $\ddagger$ Department of Cell and Molecular Biology, University of Rhode Island, Kingston RI 02881
}

E-mail: greenfield@uri.edu 


\begin{abstract}
Many classical antimicrobial peptides adopt an amphipathic helical structure at a water-membrane interface. Prior studies led to the hypothesis that a hinge near the middle of a helical peptide plays an important role in facilitating peptide-membrane interactions. Here, dynamics and vibrations of a designed hybrid antimicrobial peptide LM7-2 in solution were simulated to investigate its hinge formation. Molecular dynamics simulation results on the basis of the CHARMM36 force field showed that the $\alpha$-helix LM7-2 bent around two or three residues near the middle of the peptide, stayed in a helix-hinge-helix conformation for a short period of time, and then returned to a helical conformation. High resolution computational vibrational techniques were applied on the LM7-2 system when it has $\alpha$-helical and helix-hinge-helix conformations to understand how this structural change affects its inherent vibrations. These studies concentrated on the calculation of frequencies that correspond to backbone amide bands I, II, and III: vibrational modes that are sensitive to changes in the secondary structure of peptides and proteins. To that end, Fourier transforms were applied to thermal fluctuations in $\mathrm{C}-\mathrm{N}-\mathrm{H}$ angles, $\mathrm{C}-\mathrm{N}$ bond lengths, and $\mathrm{C}=\mathrm{O}$ bond lengths of each amide group. In addition, instantaneous all-atom normal mode analysis was applied to monitor and detect the characteristic amide bands of each amide group within LM7-2 during the MD simulation. Computational vibrational results indicate that shapes and frequencies of amide bands II and especially III were altered only for amide groups near the hinge. These methods provide high resolution vibrational information that can complement spectroscopic vibrational studies. They assist in interpreting spectra of similar systems and suggest a marker for the presence of the helix-hinge-helix motif. Moreover, radial distribution functions indicated an increase in the probability of hydrogen bonding between water and a hydrogen atom connected to nitrogen $(\mathrm{HN})$ in such a hinge. The probability of intramolecular hydrogen bond formation between HN and an amide group oxygen atom within LM7-2 was lower around the hinge. No correlation has been found between the presence of a hinge and hydrogen bonds between amide group oxygen atoms and the hydrogen atoms of water molecules. This result
\end{abstract}


suggests a mechanism for hinge formation wherein hydrogen bonds to oxygen atoms of water replace intramolecular hydrogen bonds as the peptide backbone folds.

\section{Introduction}

The resistance of bacteria and fungi against conventional antibiotics is increasing. ${ }^{1-4}$ Studies show future potential of Anti-Microbial Peptides (AMPs) as applicable antibiotic drugs based on two main features: their unique mechanism of action ${ }^{5,6}$ and rapid killing of bacteria. ${ }^{6}$ The interactions between an AMP and a bacterial membrane play a crucial role in the AMP mechanism, ${ }^{1,4,7-9}$ and design principles have recently been reviewed. ${ }^{10}$ Although several experimental and computational studies have tried to propose models to explain AMPs mechanisms of action and have explored some details of AMP-membrane interactions, ${ }^{3,11-13}$ there is still much uncertainty in why and how these interactions occur. . $^{8,12,14}$

AMPs that disrupt a bacterial membrane generally take the shape of an $\alpha$-helix. ${ }^{11}$ Some helical AMPs have a potential to bend into a helix-hinge-helix conformation when next to a lipid membrane ${ }^{15,16}$ or when dissolved in a membrane-mimicking solvent. ${ }^{17,18}$ Some studies have led to the hypothesis that a flexible loop or hinge in the middle of a helical peptide increases its antibacterial activity possibly by allowing either or both of the C-and N-terminal groups to be bound to the membrane, ${ }^{18,19}$ which consequently facilitates the process of the peptide penetrating into lipid bilayers. ${ }^{16}$ A flexible hinge structure in the middle of the peptide can also contribute to the C-terminal group spanning the lipid bilayer. ${ }^{20}$

Potential for a hinge to form, within the ensemble of conformations available to the peptide, is related to the amino acid sequence. Proline is the most common amino acid known to promote $\beta$-turns, ${ }^{21}$ which can initiate formation of a hinged region, and studies have demonstrated the key role of proline in the antibiotic activities of many AMPs. ${ }^{17,18,20,22,23}$ It has been observed that sequences such as Lys-Phe-Gly-Arg, ${ }^{15}$ Val-Gly, ${ }^{15,24}$ Gly-Ile-Gly, ${ }^{20}$ and His-His-Leu-Ala ${ }^{16}$ in the middle of different AMPs formed hinged regions that led to 
better AMP-membrane binding or AMP insertion into the target membranes.

Although most studies on helix-hinge-helix AMPs have demonstrated that existence of a hinged region plays a crucial role in AMP antibacterial activity, other studies indicated that the existence of a hinge is not important for that activity. ${ }^{25,26}$ For example, a molecular dynamics study of Cecropin B-membrane interactions suggested that the flexible hinge region (Ala22-Gly23-Pro24) contributes to the insertion of Cecropin B into the membrane by either or both terminal groups. ${ }^{19}$ However, Wang et al. demonstrated experimentally that deleting this hinge did not affect AMP antimicrobial activity. ${ }^{26}$ The molecular dynamics study of Sun et al. ${ }^{25}$ demonstrated that activity of melittin as an antimicrobial peptide was retained when Pro was replaced with Ala. They indicated that a hinge region around Pro does not play the key role in the antibacterial activity of melittin. ${ }^{25}$ Therefore, the mechanism of action of helix-hinge-helix-capable anti-microbial peptides has not been fully understood yet. ${ }^{23}$

It would be desirable to monitor changes in the secondary structure of AMPs. Experimental methods such as crystallographic analysis, NMR, circular dichroism, and vibrational spectroscopy techniques have been used to study the structures and conformations of proteins and peptides, including AMPs. ${ }^{27-33}$ Among these methods, NMR is the most detailed method for studying the structure of AMP-membrane interactions in solution. However, a high peptide concentration is needed for NMR studies, which can lead to possible protein or peptide aggregation. In addition, NMR can only probe protein or peptide structures and measure peptide dynamics over time scales longer than $\mu s$. Hence, short-time kinetic studies are difficult to carry out by NMR. Although vibrational studies provide low-resolution structural information compared to NMR, they can provide insight into unfolded and folded peptide conformational changes, such as in the secondary structure, over quite a range of time scales (from fs to s) and in either dilute solution or the presence of a membrane. ${ }^{30,32,34-41}$

Several bands measured via vibrational spectroscopy can be used for analyzing protein and peptide structures. The most characterized and commonly used vibrational modes to probe protein secondary structure are the backbone amide bands of the polypeptide. Amide 
I (AmI, around $1600-1700 \mathrm{~cm}^{-1}$ ) results mainly from the carbonyl $\mathrm{C}=\mathrm{O}$ stretching vibration in amide groups; amide II (AmII, around $1480-1580 \mathrm{~cm}^{-1}$ ) is an out-of-phase combination of both $\mathrm{C}-\mathrm{N}-\mathrm{H}$ in-plane bending and $\mathrm{C}-\mathrm{N}$ stretching; and amide III (AmIII, around 1230$\left.1340 \mathrm{~cm}^{-1}\right)$ is an in-phase combination of $\mathrm{C}-\mathrm{N}-\mathrm{H}$ in-plane bending and $\mathrm{C}-\mathrm{N}$ stretching. ${ }^{4-47}$ In some studies, three different frequency ranges have been considered for the AmIII band $\left(\mathrm{AmIII}_{1}, \mathrm{AmIII}_{2}\right.$, and $\left.\mathrm{AmIII}_{3}\right){ }^{37,48-51}$ In this work, we use the general phrase AmIII to refer to the $\mathrm{AmIII}_{3}$ band.

One objective of spectroscopic studies is to identify changes in conformation or local environment through a change in the spectrum. Vibrational studies such as infrared (IR) ${ }^{45}$ Raman, ${ }^{44,46,52}$ Raman optical activity (ROA), ${ }^{36,42,43,46}$ and Ultraviolet Resonance Raman (UVRR), ${ }^{41,42,46,47,53}$ demonstrate ways that amide bands are sensitive to the secondary structure of proteins. Lednev et al. probed thermal unfolding of an $\alpha$-helical peptide by comparing amide UVRR bands over different temperatures. ${ }^{34}$ Some UVRR studies demonstrated that the AmIII band is sensitive to the peptide secondary structure ${ }^{35,54}$ with a vibrational frequency that depends mostly on $\psi$ and less on the $\varphi$ angle of a peptide. ${ }^{35,38,54}$ Oladepo et al. distinguished between $\alpha$-helix-like states such as $\pi$ and $3_{10}$ by monitoring the $\mathrm{AmIII}_{3}$ and $\mathrm{C}_{\alpha}-\mathrm{H}$ UVRR vibrational frequencies and their dependence on peptide bond $\psi$ angles. ${ }^{40}$ UVRR studies pointed out the frequency dependence of amide bands on hydrogen bonding of amide groups of a peptide with water or with other peptide amide groups. ${ }^{38}$ Fourier Transform IR (FTIR) results showed frequency differences of the AmI band and especially the AmIII region among an $\alpha$-helix, a $\beta$-sheet, and a random coil protein, which helped to predict protein secondary structure from the IR spectrum. ${ }^{55}$ Schweitzer-Stenner et al. studied AmIII bands for diverse dipeptides by combining polarized Raman spectra, FTIR spectra, and quantum calculations, and they showed the dependence of AmIII on torsion angle $\psi$. They also demonstrated interaction of AmIII bands with side chain vibrations. ${ }^{56}$ Off-diagonal peaks in two-dimensional infrared (2D IR) spectroscopy of peptides arose due to anharmonic overlaps between AmI vibrations. Peak positions and shapes enabled distin- 
guishing $\alpha$-helical and $3{ }_{10}$-helical configurations. ${ }^{33,57,58}$

Several studies that have been done on AMPs contributed to better understanding of AMP-membrane interactions. ${ }^{59}$ For instance, UVRR was applied on three different AMPs in two different unfolded and fully folded ( $\alpha$-helix) environments and also in the presence of zwitterionic lipids. Comparing these spectra helped to find the helical percentage of each AMP when they were bound to lipids. ${ }^{60}$ Isotope labeling of specific amide groups with ${ }^{13} \mathrm{C}={ }^{18} \mathrm{O}$ has enabled 2D IR results to distinguish which amide groups of an AMP were immersed in more polar vs less polar regions of a lipid bilayer. ${ }^{32}$

Computational methods invoke various approaches to predict a vibrational spectrum. All-atom normal mode analysis (NMA) obtains amide vibrational bands from an optimized (minimum energy) structure within a harmonic approximation. ${ }^{56,61-64}$ Some theoretical studies have focused on amide vibrational bands of N-methylacetamide, as a simple amide group, using gas phase or solution ab initio calculations ${ }^{61}$ that are sometimes aided by estimates from molecular dynamics (MD) of solvent locations. ${ }^{62}$ The structural sensitivity of AmIII was studied for diverse isolated dipeptides by DFT. ${ }^{56}$ Peptide conformation and solvent environment can serve as inputs to such calculations.

Some theoretical studies have considered peptides larger than a few amino acids. Hahn studied amide bands of poly-L-alanine peptides with two to five residues in assorted, welldefined secondary structures. Normal mode results of ab initio calculations were used to investigate local vibrational amide modes through all these poly-L-alanine peptides. ${ }^{64}$ Yamamoto et al. computed the basis of ROA spectra by DFT for hydrated and unhydrated $\alpha$-helical (Ala) 18 . They proposed that changes in solvent environment rather than in protein conformation are the main reason for intensity changes of the AmIII band. ${ }^{65}$ Mensch et al. studied the conformational dependency of amide bands of poly-L-alanine by a combination of Raman and ROA spectra and quantum calculations. They developed a database that helps to interpret ROA patterns of different proteins. ${ }^{66}$

Peptide conformation fluctuates around its stable structure in a realistic system, ${ }^{56}$ and 
spectra obtained by experimental vibrational techniques are averages over many molecule conformations in solution. Some studies calculated overall averaged vibrational spectra by applying a combination of Raman and ROA spectra with computational methods. ${ }^{67,68}$ For example, Furuta et al. ${ }^{67}$ have done a computational study on a tetra-L-alanine in solution in addition to experimental Raman and ROA. The tetra-L-alanine solution was heated gradually in a classical MD simulation, quantum mechanical/molecular mechanical ONIOM calculations were applied on $250 \mathrm{MD}$ snapshots to simulate the overall ROA spectra, and then the overall averaged harmonic vibrational spectra were calculated and used for vibrational studies. On the basis of this study, a marker for poly-L-proline II helix type conformation was proposed. ${ }^{67}$

Vibrational spectra can also be determined using configurations from classical MD simulations with a vibrational frequency map, summarized in a recent review. ${ }^{69}$ For example, Woys et al. used solvent positions from MD to calculate electric fields; then they computed AmI absorption frequencies using a linear correlation. ${ }^{32}$ Malolepsza and Straub developed two correlations between (carbon, nitrogen, oxygen) positions and AmI IR peaks. ${ }^{70}$ Cunha et al. ${ }^{71}$ compared several methods for determining local electrostatic fields and coupling models on the basis of protein conformations from MD simulations that used different force fields. AmI peaks needed to be shifted in each case in order to match experimental 1D or 2D IR data.

Instantaneous NMA refers to computing vibrational modes for molecules along a sequence of configurations, regardless of whether or not each configuration is at a local potential energy minimum. Applying instantaneous NMA on the results of MD simulations can imitate the realistic vibrational spectra of molecules although it is an expensive computational method. ${ }^{72}$ Some studies have implemented instantaneous NMA on small systems, which included only one amide group. Farag and Bastida applied this method through Born-Oppenheimer MD simulation to study the AmI vibrational relaxation mode of $\mathrm{N}$ methylacetamide-D. ${ }^{63}$ Instantaneous NMA was used to study vibrational relaxation of the 
AmI mode of $\mathrm{N}$-methylacetamide- $\mathrm{D}^{73}$ and alanine dipeptide ${ }^{74}$ in solution. In a previous computational study, we applied instantaneous NMA on systems of tryptophan and tyrosine in solution and studied out-of-plane vibrations of atoms in the rings of these aromatic amino $\operatorname{acids.~}^{75}$

The vibrational spectrum of a peptide system is a combination of all possible vibrational modes, and several similar modes can overlap around similar frequencies. ${ }^{36,41,44}$ Such a spectrum of the whole molecule is quite complex. The hypothesis of this work is that if we can study the vibrational spectrum locally around a specific part of a protein or peptide in more detail, then more information about the local conformational dependency of amide bands can be revealed.

Experimental vibrational techniques can be a suitable tool for monitoring the process of the hinge formation in peptide because of amide band sensitivity to backbone conformation, especially when the process is fast. But these experimental tools provide low resolution structural information about the peptide, and interpreting the complex spectra of a peptide has been challenging. Amide bands obtained by experimental vibrational tools are a summation of coupled modes of all amide bands through the peptide. ${ }^{36,41,44}$ Contributions of individual amide groups have been identified through isotope studies, which create separable peaks for the altered amide group. ${ }^{32,76}$ In the absence of such significant chemical synthesis efforts, it is very challenging to investigate changes of amide bands because of small changes in the conformation of a subset of peptides for a short time.

The present work focuses on conformations of a hybrid AMP LM7-2, which was designed using combinations of naturally occurring Dermaseptin and Pleurocidin AMPs by Martin's group. ${ }^{77}$ LM7-2 is similar to the P-DER AMP of Hancock and co-workers. ${ }^{78}$ LM7-2 has 27 amino acids (H-ALWKTMLKKAAHVGKHVGKAALTHYLN-NH2) with an N-terminal amide. Ryder and Martin stated that this peptide has an amphipathic $\alpha$-helical structure on a water-cell membrane surface. ${ }^{77}$ By using the Chou-Fasman algorithm, ${ }^{79}$ they predicted that LM7-2 has a noteworthy hinge region. LM7-2 has an acceptable activity against four 
bacteria: E. coli and P. aeruinosa as gram-negative bacteria, and E. faecalis and S. aureus as gram-positive bacteria. ${ }^{77}$

In this work, we seek to apply statistical sampling over thermal fluctuations while also noting frequency changes that arise when one of many similar functional groups undergoes conformational change. To imitate the realistic vibrational behavior of molecules and to study local vibrational spectra in more detail, we apply instantaneous NMA using the results of molecular dynamics (MD) simulations of LM7-2 fluctuations about an $\alpha$-helical conformation in solution.

First, we studied the geometry of the peptide to recognize occasional presence of a hinge. Then we applied computational vibrational methods of Fourier transform and instantaneous NMA to understand how these spontaneous changes in the peptide conformation affect peptide vibrations. We investigated these complex amide bands at high structural resolution during short time periods and determined differences in vibrations between the helix and hinge states of LM7-2. By implementing these methods, we locally studied amide bands of each amide group through LM7-2 in extended detail, and we quantified local changes in amide bonds between the helical and the hinge conformations.

Our results demonstrate a relation between the hinge formation and local amide band shifts, and we identify amide band frequency shift markers for the temporary transformation of an $\alpha$-helix to a helix-hinge-helix conformation. Intermolecular and intramolecular hydrogen bonding of LM7-2 around the hinge were also studied. Our results contribute to interpretation of vibrational techniques for monitoring folding and unfolding processes of peptides in similar systems. 


\section{Methodology}

\section{Simulations}

An $\alpha$-helical structure of LM7-2 was built in VMD $^{80}$ software by using the Molefacture plugin. Default $\alpha$-helix torsion angles of $\varphi=-57^{\circ}$ and $\psi=-47^{\circ}$ were chosen for all residues of the peptide; torsion angles of $0^{\circ}$ correspond to cis. LM7-2 has an amidated C-terminus and protonated N-terminus. On the basis of the experimental environment, ${ }^{77}$ both nitrogen atoms of all histidine (His) rings and the amine group of all lysines (Lys) were protonated for the simulation. Hence, LM7-2 has 9 positive charges in total. LM7-2 was solvated with 31784 water molecules in a box with an initial dimension of $100 \times 100 \times 100 \AA^{3}$ by VMD software. ${ }^{80}$ Since the desired $\mathrm{NaCl}$ concentration was $0.15 \mathrm{M}$ according to the experimental condition, ${ }^{77} 87 \mathrm{Na}^{+}$and $96 \mathrm{Cl}^{-}$ions were added to the system, which made the $\mathrm{Na}^{+}$and $\mathrm{Cl}^{-}$concentrations around $0.145 \mathrm{M}$ and $0.152 \mathrm{M}$ in solution.

The NAMD package ${ }^{81}$ was used for minimization and MD simulation. At first, 5000 steps of conjugate gradient energy minimization were applied on the system. Then, $100 \mathrm{~ns}$ of NPT MD simulations were performed on the system with a time step of 1 fs. The first $30 \mathrm{ps}$ of this time period were considered for equilibration. The CHARMM36 ${ }^{82-84}$ force field was implemented for minimization and MD simulation. The TIP3P model ${ }^{85}$ was used for water molecules. Three-dimensional periodic boundary conditions were applied. For nonbonded interactions, neighbor lists were updated every 10 steps, the cutoff was $12 \AA$, the switch distance was $10 \AA$, and a pair list distance was $14 \AA$ for all simulations. Particle mesh Ewald $^{86}$ was used for long range electrostatic interactions. To maintain the temperature at $310 \mathrm{~K}$ and pressure at $1 \mathrm{~atm}$, Langevin dynamics with a damping coefficient of $100 \mathrm{fs}$ and Nose-Hoover Langevin piston pressure control with barostat oscillation and damping time scales of 200 fs and 100 fs were used. ${ }^{87,88}$ Conformations were saved in pdb format every 100 fs. Collective Variables Module ${ }^{89}$ (colvar) of NAMD was used to store bond lengths, bond angles, and backbone torsion angles every 1 fs over 100 ns of MD simulation for peptide 
vibrational analysis.

\section{Vibrational Analysis}

Fourier Transform method. Applying Fourier transformation (FT) to time-dependent internal coordinates $(\mathrm{C}=\mathrm{O}$ and $\mathrm{C}-\mathrm{N}$ bond lengths and $\mathrm{C}-\mathrm{N}-\mathrm{H}$ angles of each amide group in LM7-2) converts the time domain into the frequency domain and provides their vibrational frequencies. To that end, we used the Octave package ${ }^{90}$ for fast FT (FFT). As we explain in the results section, LM7-2 experienced helical and helix-hinge-helix conformations. Hence, we applied FFT on $\mathrm{C}=\mathrm{O}$ and $\mathrm{C}-\mathrm{N}$ bond lengths and $\mathrm{C}-\mathrm{N}-\mathrm{H}$ angle of all amide groups of LM7-2 for four selected 200 ps time periods in which LM7-2 has either a helical or a helixhinge-helix conformation. Wavenumbers computed from FFT spanned $n /[c N \delta t]=0.167$ to $33400 \mathrm{~cm}^{-1}$ for $n=1$ to $N=2 \times 10^{5}$ steps, and $c$ indicates the speed of light. These

calculated vibrational spectra over the more narrow range 1100 to $1800 \mathrm{~cm}^{-1}$ enable us to compare amide band vibrations among these states of LM7-2.

All-atom normal mode analysis. We implemented all-atom normal mode analysis (NMA) as another method for vibrational studies. NMA provides all vibrational frequencies of a system within a harmonic approximation, and atom vibrational motions correspond to each frequency ("normal modes"). Hence, we could investigate details of vibrational motions of LM7-2 that go beyond information available from the FT method. We explained our implementation of NMA previously. ${ }^{75}$ Briefly, we calculate analytically the Hessian matrix $(\boldsymbol{H})$, which is the second derivative of potential energy with respect to Cartesian coordinates. A force-constant matrix $(\boldsymbol{F})$ is calculated by

$$
\boldsymbol{F}=\boldsymbol{M}^{-1 / 2} \boldsymbol{H} \boldsymbol{M}^{-1 / 2}
$$


where $\boldsymbol{M}$ is a diagonal matrix containing the atomic masses. Then, we solve

$$
\boldsymbol{F} \boldsymbol{V}_{i}=\lambda_{i} \boldsymbol{V}_{i}
$$

so all eigenvalues $\lambda_{i}$ and their orthonormal eigenvectors $\boldsymbol{V}_{i}$ are calculated. By assuming an oscillatory solution to the equations of motion, vibrational frequencies $\left(\nu_{i}\right)$

$$
\nu_{i}=\sqrt{\lambda}_{i} / 2 \pi
$$

are obtained. If $\mathrm{N}$ is the number of atoms, there are $3 \mathrm{~N}$ frequencies $\left(\nu_{i}\right)$ and $3 \mathrm{~N}$ sets of normal vectors $\left(\boldsymbol{V}_{i}\right)$; each normal vector specifies vibrational movements of atoms that correspond to its frequency. Since six calculated eigenvalues are zero, which represent translation and rotation of the system, there are $3 \mathrm{~N}-6$ vibrational modes in total.

We modified our previously developed NMA package ${ }^{75}$ for the current study. In this version, to accelerate solving eigenvalue-eigenvector problems numerically, cuSOLVER of nVIDIA CUDA toolkits ${ }^{91}$ was used instead of the $\mathrm{C}++$ LAPACK library. ${ }^{92}$ Potential energy $\varphi$ and $\psi$ crossterms were included in addition to the expressions for all bonds, angles, torsions, improper torsions, Urey-Bradley, and nonbonded (Lennard-Jones and Coulomb) interactions for building the Hessian matrix. CHARMM36 considers $\varphi$ and $\psi$ crossterm extension potential energies for the peptide backbone. ${ }^{93,94}$ As we explained previously, ${ }^{75}$ we neglected the long range electrostatic interactions in NMA because the second derivatives of this potential energy term are close to zero. We implemented the same cutoff and switch functions and the same potential energy equations that are defined in NAMD ${ }^{81}$ software. To use the same force field parameters for building the Hessian matrix, we amended the NAMD 81 source code to export all force field parameters at the beginning of an MD simulation. The Hessian matrix was calculated analytically except for crossterm potentials. NAMD ${ }^{81}$ uses CMAP for crossterm calculations and calculates the first derivatives of the crossterm potential energy with respect to $\varphi$ and $\psi$ numerically. Hence, we amended the NAMD ${ }^{81}$ source 
code to calculate the second derivatives and mixed derivatives of the crossterm potential energy with respect to $\varphi$ and $\psi$. These calculated derivatives were generated and stored by the amended NAMD ${ }^{81}$ for each MD step and were read to build the Hessian matrix.

Among all vibrational modes of LM7-2 that were obtained by NMA, we are interested in amide group vibrations: AmI, AmII, and AmIII. As explained in the introduction, these amide bands are sensitive to the secondary structure of the peptide. ${ }^{30,36,41-47,52,53}$ To find these specific modes, orthonormal eigenvectors of these three modes are needed. Hence, we designed an alanine (Ala) dipeptide (AA) as a reference molecule to find these three modes.

An AA dipeptide with neutral amine N-terminus and neutral carboxyl C-terminus was built in Avogadro software. ${ }^{95}$ The automatic psf builder of $\mathrm{VMD}^{80}$ software was used to create force field parameters on the basis of the CHARMM36 ${ }^{82-84}$ force field.

The AA dipeptide was minimized in vacuum and NMA was applied. Our NMA package supplied an animation of each mode that can be viewed by $\mathrm{VMD}^{80}$ software. By watching animations, we determined three reference eigenvectors that represented AmI, AmII, and AmIII vibrational modes. To investigate the effects of end group type and charge on amide bands, NMA was applied on two alternative structures: minimized helical AA with a carboxylate $\left(\mathrm{COO}^{-}\right)$and a protonated amine $\left(\mathrm{NH}_{3}^{+}\right)$, and minimized helical AA with methylamide terminal groups. Modes were compared with those of the amine-acid case.

Backbone torsion angles of minimized structures of the AA dipeptide deviated from $\alpha$ helix torsion angles. To confirm that changes in backbone torsion angles $\varphi$ and $\psi$ only affect amide band frequencies and that amide band vibrational movements are independent of these changes, NMA was implemented on neutral and zwitterionic AA dipeptides with torsion angles of $\varphi=-57^{\circ}$ and $\psi=-47^{\circ}$. These dipeptides were built in $\operatorname{VMD}^{80}$ software by using the Molefacture plugin.

Instantaneous normal mode analysis. For LM7-2, NMA was applied over the same four selected 200 ps time periods that exhibited helical or helix-hinge-helix conformations. In this instantaneous NMA approach, we analyzed all conformations within 2000 MD steps (pdb 
files) that were stored for each time period. There are 95980 atoms in the system; therefore, the Hessian matrix has a size of $N^{2} \sim(95980 \times 3)^{2}$. Although our package could analytically build this large Hessian matrix very quickly, calculating eigenvalues and eigenvectors is expensive because these calculations scale as $\sim N^{3}$. On the other hand, we cannot apply instantaneous NMA only on atoms of the peptide and omit ions and water molecules. Water molecules in the neighborhood of amine and carbonyl groups of the peptide affect vibrational frequencies. ${ }^{38}$ Hence, water molecules and ions whose atoms are closer than $4 \AA$ to nitrogen and oxygen atoms of all LM7-2 amide groups were considered for instantaneous NMA.

There are 26 amide groups between residues of LM7-2, and each amide group has its own amide bands. Because these vibration calculations are performed on detailed configurations from a molecular dynamics simulation, computations enable amide bands of each amide group in a single molecule to be distinguished among all vibrational frequencies obtained by instantaneous NMA.

Normal modes within instantaneous NMA. To monitor distinct amide group contributions and to assign labels for all amide bands within the peptide, a dot product technique was implemented for each MD step (pdb file). A flowchart that explains this method is provided in Figure S1. First, the minimized reference AA was aligned on each amide group of LM7-2. Carbon $(\mathrm{C})$, oxygen $(\mathrm{O})$, and nitrogen $(\mathrm{N})$ atoms in the amide group of the reference and the amide groups of LM7-2 were used as basis points for this alignment, and orthonormal eigenvectors were oriented accordingly. As explained earlier, there is an eigenvector set for each amide band. Atoms $\mathrm{C}, \mathrm{N}$, and $\mathrm{O}$ of amide groups are involved in AmI bands, and C, N, and hydrogen (HN) connected to $\mathrm{N}$ of amide groups are involved in AmII and AmIII bands. Hence, we reduced the eigenvectors of reference modes by keeping ( $\mathrm{x}, \mathrm{y}, \mathrm{z})$ components of atoms $\mathrm{C}, \mathrm{N}, \mathrm{O}$ for $\mathrm{AmI}$ and atoms $\mathrm{C}, \mathrm{N}, \mathrm{HN}$ for AmII and AmIII and removing components of all other atoms. For each current snapshot of the LM7-2 system and for each studied amide group of LM7-2, we reduced all orthonormal eigenvectors by this same method. The magnitudes of remaining components of the LM7-2 eigenvectors were calculated and named 
a "participant fraction" for the specific amide band. These participant fractions were used later during final averaging. Then, the reduced eigenvectors of the reference molecule and the LM7-2, which now have the same number of elements, were renormalized. To find the most similar vibrational amide band for the current studied amide group, the dot products of the reduced reference eigenvector with all reduced eigenvectors of the LM7-2 system were calculated. If a dot product was more than 0.9, the corresponding mode and frequency were saved because vectors with a greater dot product are more similar. These analyses of instantaneous NMA calculations were repeated for all amide groups of LM7-2 to find amide band frequencies of each amide group and were repeated for all studied pdb files to calculate an arithmetic average of amide band frequencies of each amide group through LM7-2 during each time period. The averaging method is explained more easily in the context of results for the normal modes of individual amide groups. Thus the details of the averaging process are described in the next section.

\section{Results and Discussion}

\section{Geometric Analysis}

MD simulations were performed on LM7-2 in solution to sample its conformation fluctuations. Temperature, total energy, and volume fluctuations equilibrated after approximately 30 ps. Torsion angles $\varphi$ and $\psi$ were calculated throughout the peptide at each fs. Figures 1a and $1 \mathrm{~b}$ provide a heatmap of calculated torsion angles $\varphi$ and $\psi$, respectively, over $100 \mathrm{~ns}$ MD simulations. The y axis of each graph shows the position of a calculated torsion angle through the peptide. While $\mathrm{VMD}^{80}$ employs defaults of $-57^{\circ}$ and $-47^{\circ}$, Ulo et al. report that $\varphi$ and $\psi$ are $-64 \pm 7^{\circ}$ (deep teal color) and $-41 \pm 7^{\circ}$ (light green color), respectfully,

for all residues of an $\alpha$-helical peptide. ${ }^{96}$ As shown in Figures 1a and 1b, torsion angles $\varphi$ and $\psi$ of LM7-2 are mostly within these $\alpha$-helix criteria over 100 ns MD simulation, which indicates that LM7-2 experienced a helical conformation most of the time. There are some 


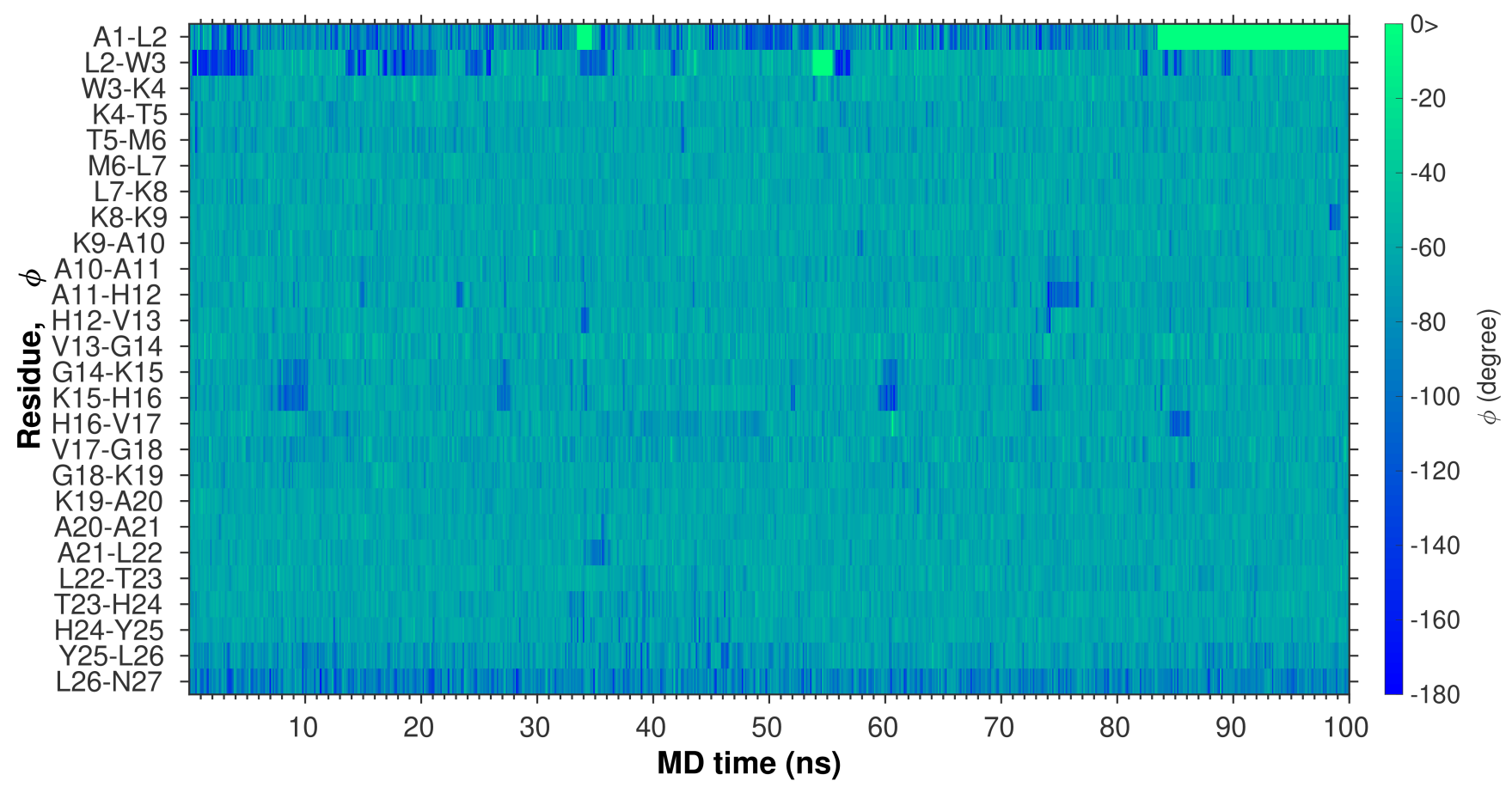

(a)

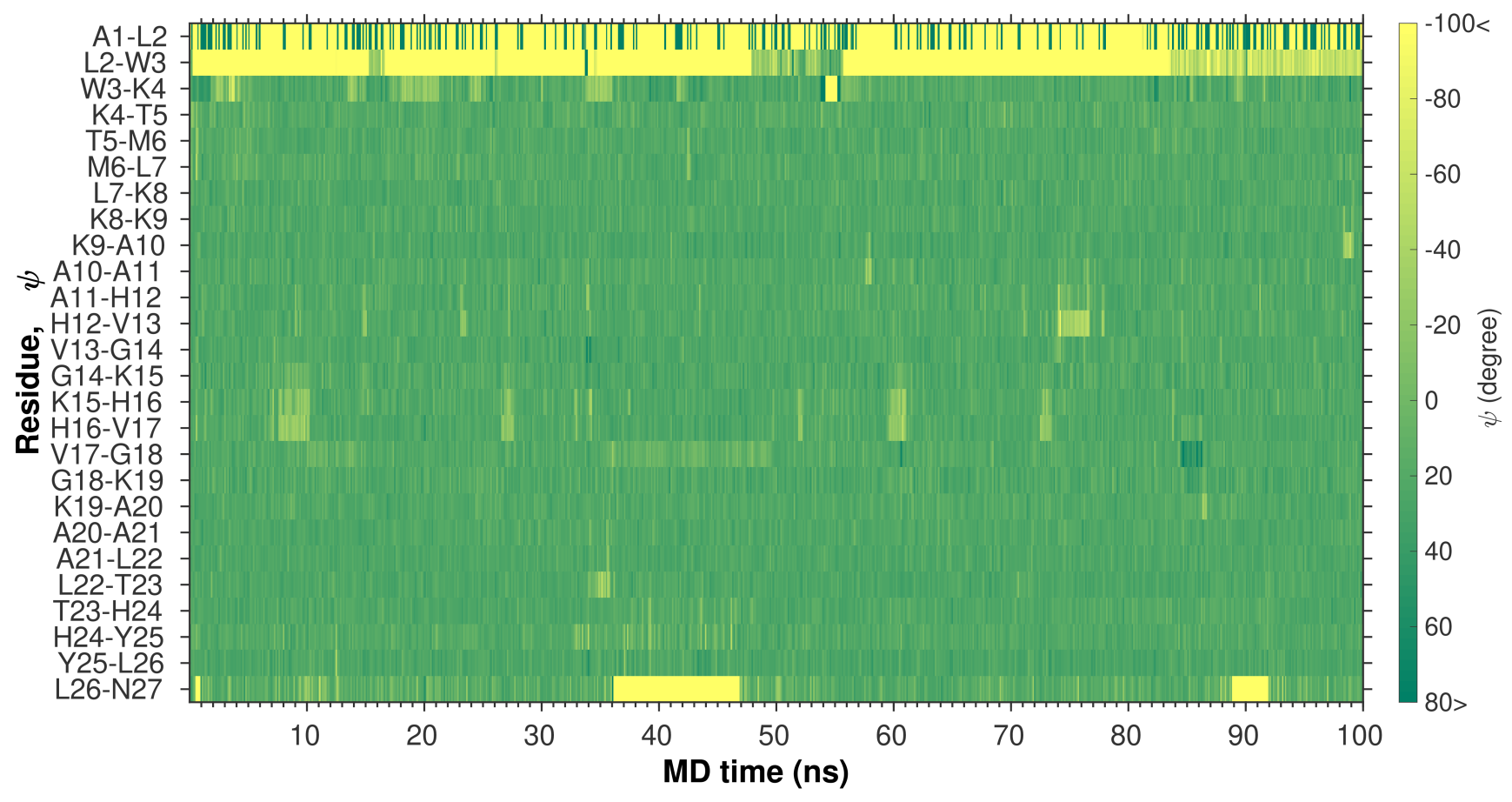

(b)

Figure 1: Heatmap of (a) $\varphi$ and (b) $\psi$ for all residues of LM7-2 over 100 ns MD simulation. A helical peptide has $\varphi=-64 \pm 7^{\circ}$ and $\psi=-41 \pm 7^{\circ} .{ }^{96}$ 

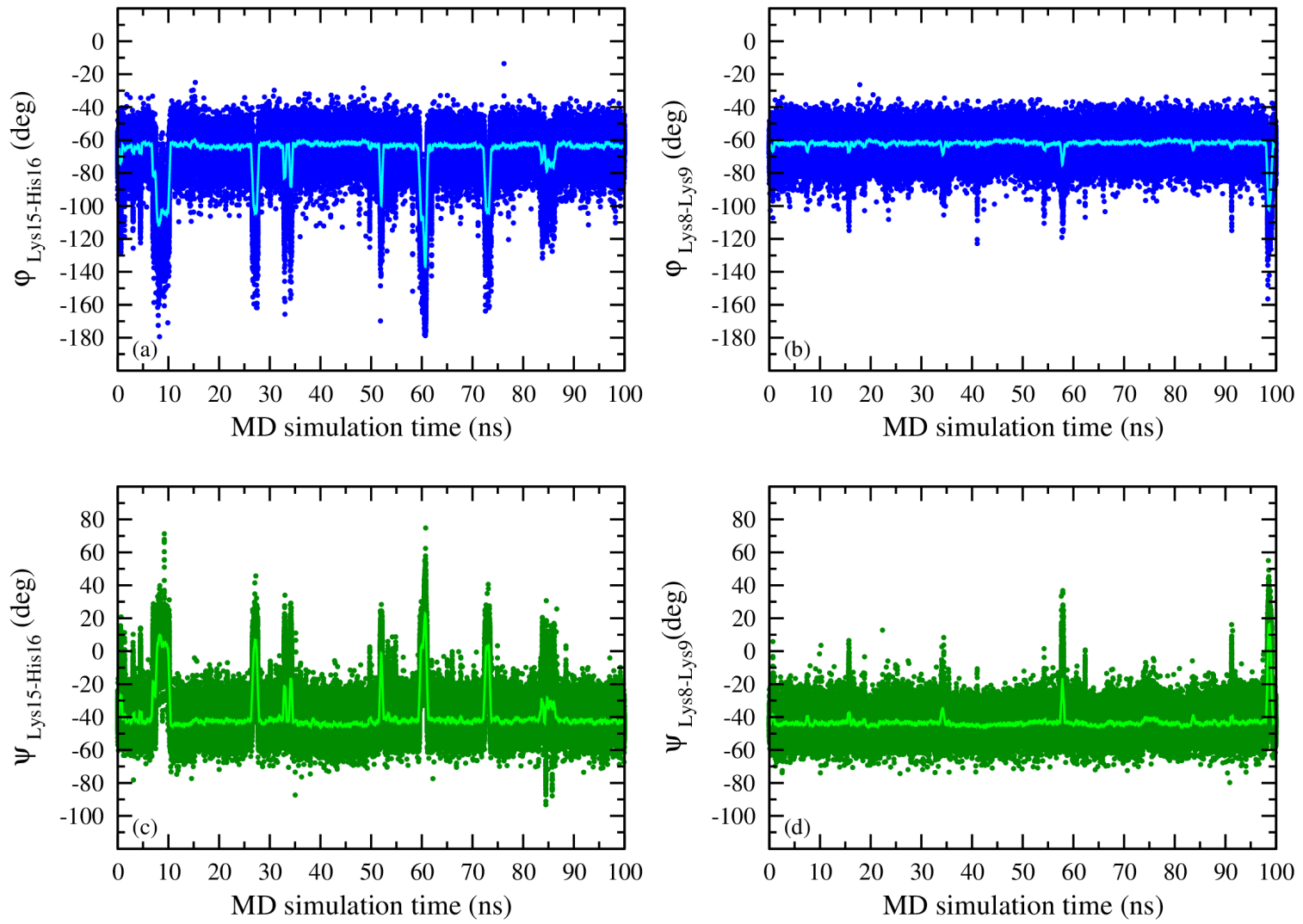

Figure 2: Torsion angles $\varphi$ of (a) Lys15-His16 and (b) Lys8-Lys9 and $\psi$ of (c) Lys15-His16 and (d) Lys8-Lys9 as a function of simulation time. A 1000-point moving average is shown in $(a, b)$ light blue and $(c, d)$ light green.

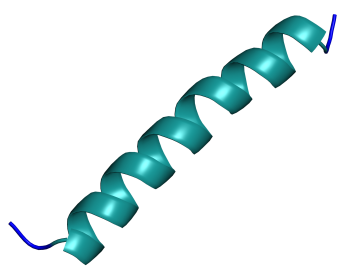

(a)

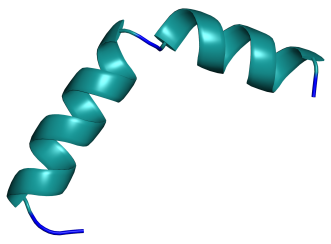

(b)

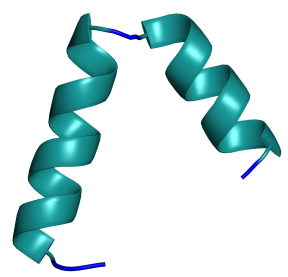

(c)

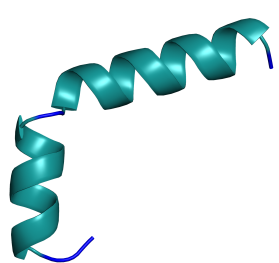

(d)

Figure 3: LM7-2 has (a) a helical conformation at $\sim 40 \mathrm{~ns}$, (b) a helix-hinge-helix at $\sim 9.5 \mathrm{~ns}$ with a hinge around Lys15-Val17, (c) a helix-hinge-helix at $\sim 60.7$ ns with a hinge around Lys15-Val17, and (d) a helix-hinge-helix at $\sim 75.9$ ns with a hinge around Ala11-Val13. Deep teal and blue colors indicate helical and loop regions, respectively. 

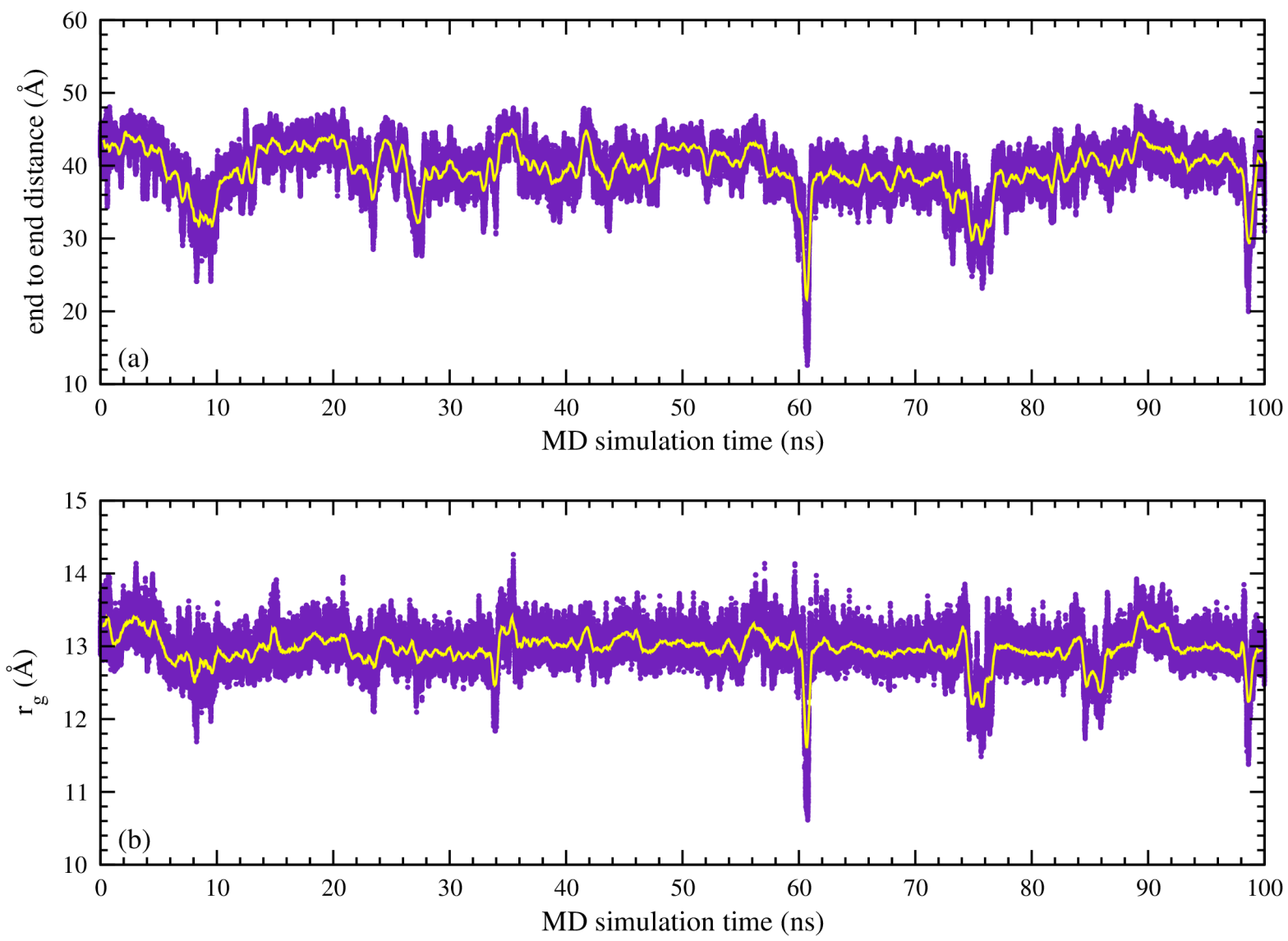

Figure 4: (a) end-to-end distance and (b) $\mathrm{r}_{\mathrm{g}}$ of a helical LM7-2 in solution over 100 ns MD simulation. A 1000-point moving average of both graphs are shown by the yellow color.

blue regions in the $\varphi$-map or light green to yellow colored regions in the $\psi$-map around residues Lys15-Val17 at 9, 27, and 60 ns, and around residue Ala11-Val13 at 75 ns.

The color changes in Figure 1 show that their corresponding torsion angles $\varphi$ and $\psi$ deviate from the $\alpha$-helix criteria, while $\varphi$ and $\psi$ of other residues remain in the $\alpha$-helix range. As an example, we provide Figure 2 to show calculated torsion angles $\varphi$ and $\psi$ for Lys15-His16 and Lys8-Lys9 residues over 100 ns MD simulations. There are some short period of times that $\varphi$ and $\psi$ of Lys15-His16 deviated significantly from the ideal $\alpha$-helix torsion angle, while these deviations are not observed for $\varphi$ and $\psi$ of Lys8-Lys9. These results demonstrate that LM7-2 differs from a helical conformation in the Lys15-His16 position for some short periods of time.

In Figure 3, the conformation of LM7-2 is shown at some specific MD times. At many 
times such as $\sim 40$ ns (Figure 3a), LM7-2 has a helical conformation. As shown in Figure 1, torsion angles $\varphi$ and $\psi$ of all residues are in the $\alpha$-helix criteria at this time. Figures 3b and $3 \mathrm{c}$ show LM7-2 bent around Lys15-Val17 residues at $\sim 9.5$ and $\sim 60.7$ ns. $\varphi$ and $\psi$ of these residues deviated from $\alpha$-helix criteria at these time points (Figure 1). This curved geometry is called helix-hinge-helix, which means a random coil (non-helical conformation) exists in the middle of a helical peptide. As indicated in Figures 1 and 2, LM7-2 reached a helix-hinge-helix conformation around Lys15-Val17 residues and stayed in that state for a short time. Figure 3d shows LM7-2 bent around Ala11-Val13 near 75 ns. Both Figures 1a and $1 \mathrm{~b}$ demonstrate a lot of non-helical regions at both terminal groups of LM7-2. Since atoms at both ends of LM7-2 have more freedom to move, LM7-2 experienced a random coil conformation at both terminal residues. These random coil regions are shown in thin blue color in Figure 3.

End-to-end distances and radii of gyration $\left(\mathrm{r}_{\mathrm{g}}\right)$ were calculated over $100 \mathrm{~ns}$ MD simulation and are plotted in Figure 4. The distance between nitrogen atoms of terminal amide groups was used for end-to-end calculations. As shown in Figure 4a, end-to-end distance fluctuated mostly around $40 \AA$ and decreased exactly when $\varphi$ and $\psi$ deviated from the $\alpha$-helix criteria (Figure 1). The minimum end-to-end distance $(\sim 12.6 \AA)$ happened at $\sim 60.7 \mathrm{~ns}$, which means LM7-2 has the most bent conformation around Lys15-Val17 at this time (Figure 3b). Movie Hinge (Supporting Information) is an animation of the most bent hinge formation around Lys15-Val17 during 59-62 ns. Similarly, $\mathrm{r}_{\mathrm{g}}$ fluctuated around an average of $13 \AA$, and the minimum $\mathrm{r}_{\mathrm{g}}$ was at $\sim 60.7 \mathrm{~ns}$ (Figure $4 \mathrm{~b}$ ). End-to-end distance and $\mathrm{r}_{\mathrm{g}}$ results confirm that a helical LM7-2 could bend in the middle and stay in a helix-hinge-helix conformation for a short time period. This helix-hinge-helix conformation is unstable and it returns to a fully helical LM7-2. These results confirm Ryder and Martin's prediction ${ }^{77}$ about a possible hinge in LM7-2. 


\section{Vibrational Analysis}

To study and compare amide band frequencies between $\alpha$-helical and helix-hinge-helix conformations for LM7-2, we applied vibrational studies on four different 200 ps time periods: 40 to $40.2 \mathrm{~ns}\left(\tau_{1}\right)$ as a helix, 9.4 to $9.6 \mathrm{~ns}\left(\tau_{2}\right)$ and 60.6 to $60.8 \mathrm{~ns}\left(\tau_{3}\right)$ as a helix-hinge-helix with a hinge around Lys15-Val17 residues, and 75.7 to $75.9 \mathrm{~ns}\left(\tau_{4}\right)$ with a hinge around Ala11-Val13 residues. The 60.6 to 60.8 ns time period was chosen because LM7-2 has the most bent conformation in this time period. Geometries at each time step ( $1 \mathrm{fs})$ were available for vibrational studies $\left(2 \times 10^{5}\right.$ pdb files per period $)$. We chose a $200 \mathrm{ps}$ time period for vibrational studies because LM7-2 with a hinged conformation was unstable and it appeared for only a short time.

\section{Fourier Transformation Method}

Fourier Transformation (FT) was applied on $\mathrm{C}-\mathrm{N}-\mathrm{H}$ angles, $\mathrm{C}-\mathrm{N}$ distances, and $\mathrm{C}=\mathrm{O}$ distances of all 26 LM7-2 amide groups over the four selected 200 ps time periods. 100-point (0.1 ps) moving averages were applied on FT results. Figures 5, S2, and S3 provide results of $\mathrm{FT}$ on $\mathrm{C}-\mathrm{N}-\mathrm{H}$ angles, $\mathrm{C}-\mathrm{N}$ distances, and $\mathrm{C}=\mathrm{O}$ distances of all amide groups during four different time periods. The frequency-dependent magnitude of each Fourier component resembles an infrared or Raman spectrum because it indicates the vibrational contributions at each frequency. It lacks the dipole-specific factors that dictate spectroscopic selection rules. These pseudo-spectra can be computed for individual amide groups because separable angle and distance fluctuations are obtained from the simulations.

First we consider $\mathrm{C}-\mathrm{N}-\mathrm{H}$ angles, which are described as components of AmIII and AmII modes. ${ }^{42-44,47}$ As shown in Figure 5, the highest peaks for C-N-H are at $1200-1250 \mathrm{~cm}^{-1}$ and $1550-1600 \mathrm{~cm}^{-1}$ for all spectra of all time periods. According to the same literature, these peak frequencies are in the range of AmIII and AmII modes, respectively. These peaks indicate that Fourier analysis identifies AmIII and AmII frequencies. In addition, AmIII and AmII include C-N stretching. The spectra of Figure S2 were obtained by applying 
FFT on $\mathrm{C}-\mathrm{N}$ distances. Both of these amide peaks are observed though with different ratios of intensities around similar frequencies. Comparisons of absolute magnitudes between FT spectra are less meaningful because of differences in physical units (degrees or $\AA$ ).

Bond length fluctuations between $\mathrm{C}$ and $\mathrm{O}$ atoms provide the main vibration of the AmI band. Results from FFT in Figure S3 show sharp peaks for all residues around 1680$1720 \mathrm{~cm}^{-1}$, which are in the range of the AmI band according to prior studies. ${ }^{42-47}$ In both Figures 5 and $\mathrm{S} 2$, there is a low intensity peak around $1680-1720 \mathrm{~cm}^{-1}$ that belongs to AmI. Changes in $\mathrm{C}-\mathrm{N}-\mathrm{H}$ bending and $\mathrm{C}-\mathrm{N}$ stretching have a minor effect on the position of $\mathrm{C}$ and $\mathrm{O}$, and consequently they lead to very small AmI band contributions. In addition, some studies described that although AmII and AmIII are mainly combinations of $\mathrm{C}-\mathrm{N}-\mathrm{H}$ bending and $\mathrm{C}-\mathrm{N}$ stretching, a small amount of $\mathrm{R}-\mathrm{C}=\mathrm{O}$ in-plane bending also happens in these modes. ${ }^{45}$ The presence of $\mathrm{C}=\mathrm{O}$ vibrational frequencies within $\mathrm{C}-\mathrm{N}-\mathrm{H}$ angle and $\mathrm{C}-$ $\mathrm{N}$ distance fluctuations demonstrates that the classical force field CHARMM incorporates anharmonic overlaps among AmIII, AmII, and AmI normal modes.

By comparing the calculated spectra of these four time periods, we can find how small conformational changes of LM7-2 affect frequencies of amide bands. We concentrated on interpreting the spectra of residues between Thr5-Thr23. As shown in Figure 1, residues located in both terminal groups of LM7-2 experienced different random conformations during each of these four time periods. LM7-2 was covered with more water molecules at both terminal groups, and atoms of these residues fluctuated more randomly among different conformational states.

The comparisons consider differences in group- and internal-coordinate-specific contributions to AmIII, AmII, and AmI modes for helical or helix-hinge-helix conformations. All major contributions to the three amide bands, AmIII and AmII in Figures 5 and S2 and AmI in Figure S3, have the same frequencies and similar shapes for residues Met6 to Ala10 and Gly18 to Thr23 during all four selected time periods. LM7-2 around all of these residues remained in a helical conformation, and both torsion angles $\varphi$ and $\psi$ fluctuated around the 

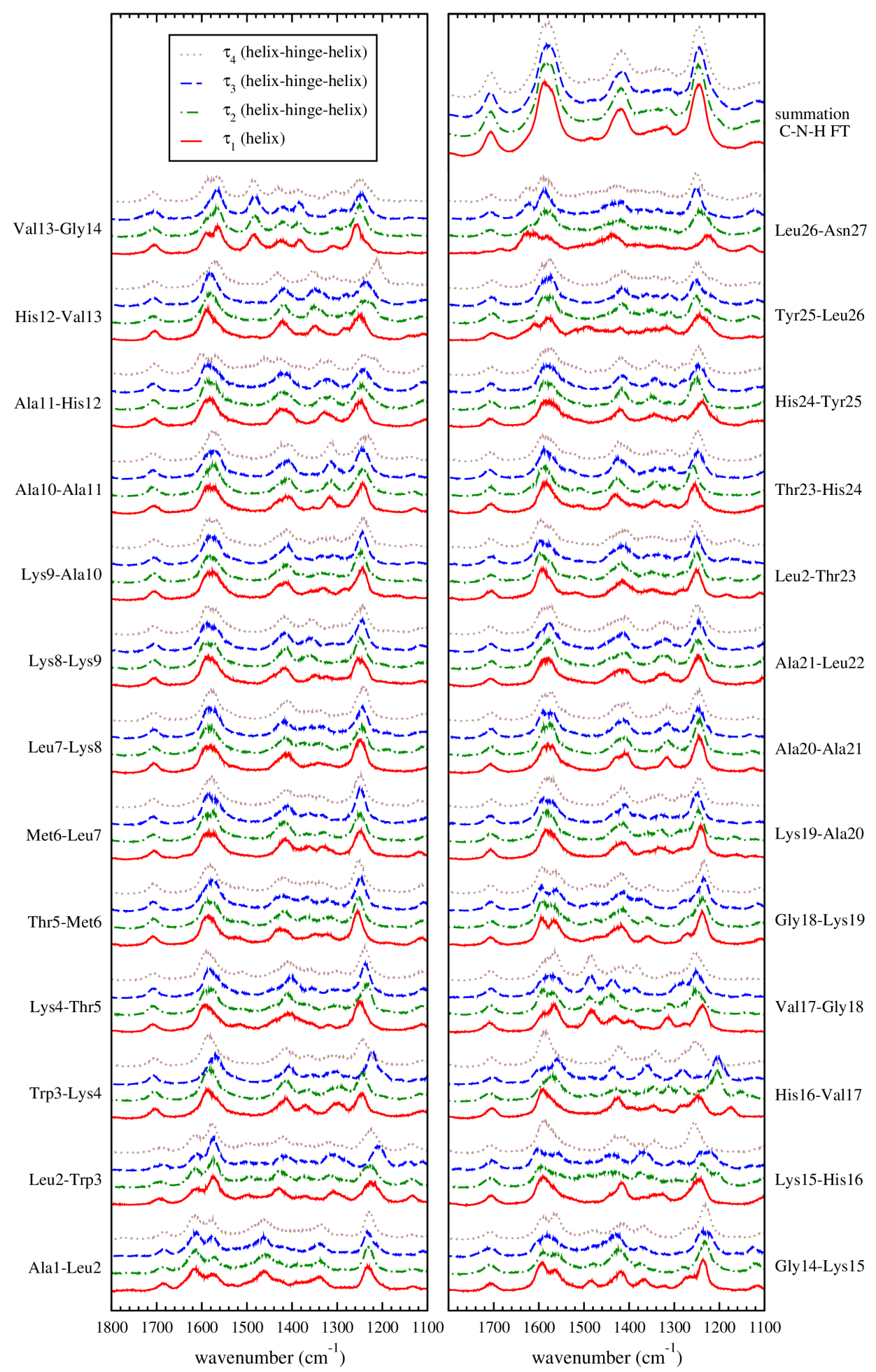

Figure 5: Vibrational spectra of amide group angle bending in LM7-2 for four selected time periods, provided by Fourier Transformation of $\mathrm{C}-\mathrm{N}-\mathrm{H}$ angle fluctuations for each amide group. Here, AmIII bands are within 1200-1250 $\mathrm{cm}^{-1}$, AmII bands are within 1550$1600 \mathrm{~cm}^{-1}$, and AmI bands are within 1680-1720 $\mathrm{cm}^{-1}$. 
$\alpha$-helix criteria (Figure 1).

On the basis of geometric analysis, LM7-2 has a hinge conformation around Ala11-Val13 during period $\tau_{4}$ and a helical conformation during other time periods. This geometric change imposes changes on vibration contributions that vary among internal coordinates. As shown in Figure 5, C-N-H contributions to AmIII bands around Ala10-Ala11 and Ala11-His12 during period $\tau_{4}$ have a different shape compared to AmIII bands of other time periods. Also, the AmIII band around His12-Val13 shifted to a lower frequency during period $\tau_{4}$ compared to the other three time periods (Figure 5 and Figure S2). The shape of $\mathrm{C}-\mathrm{N}-\mathrm{H}$ contributions to the AmII band around Ala11-His12 during period $\tau_{4}$ differs compared to AmII bands of other time periods (Figure 5 and Figure S2). In contrast to $\mathrm{C}-\mathrm{N}-\mathrm{H}$ contributions (Figure 5), in which the AmII band around His12-Val13 during period $\tau_{4}$ shifted to a lower frequency compared to other time periods, only the shape of $\mathrm{C}-\mathrm{N}$ contributions to this AmII band during period $\tau_{4}$ differs from other time periods in Figure S2. The spectra of Figure S3 show that $\mathrm{C}=\mathrm{O}$ contributions to $\mathrm{AmI}$ bands have similar shapes and frequencies around residues Ala10-Gly14 for all time periods. The helix-hinge-helix conformation of LM7-2 during period $\tau_{4}$ causes changes in pattern and frequencies of AmIII and AmII bands, which indicates the sensitivity of AmIII and AmII to the hinge around Ala10-Gly14 residues. AmI vibrations at the hinge were not impacted as much by this local change. These differences in shape and frequencies of AmIII and AmII bands can be used to detect and monitor the hinge.

LM7-2 is bent more often around Lys15-VAl17 residues. Periods $\tau_{2}$ and $\tau_{3}$ are examples of this geometry, and the most bent conformation was observed during the $\tau_{3}$ time period. Exactly around Lys15-His16 and His16-Val17, shapes and frequencies of AmIII and AmII bands differed during periods $\tau_{2}$ and $\tau_{3}$ compared to periods $\tau_{1}$ and $\tau_{4}$, when LM7-2 has a helical conformation around Lys15-His16 and His16-Val17 residues (Figure 5). Less intense and broader peaks were observed for AmIII bands around Lys15-His16 during periods $\tau_{2}$ and $\tau_{3}$. AmIII bands also shifted to lower frequencies around Lys15-His16 during periods $\tau_{2}$ and $\tau_{3}$, and the largest shift was around His16-Val17 (Figure 5 and S2). The AmII band 
of Lys15-His16 shows two peaks during period $\tau_{3}$ (the most bent one) with one shifted to a higher and another one shifted to a lower frequency compared to other time periods. During period $\tau_{2}$, the AmII band shifted slightly to a higher frequency around Lys15-His16 (Figure 5 and S2), and there is a slight shoulder in the right hand side of the peak (Figure 5). As shown in Figure 5, AmII band of His16-Val17 shifted to a lower frequency during time period $\tau_{3}$, and there is a shoulder in the left hand side of the peak. This shoulder indicates the possible existence of another peak that has a higher frequency compared to periods $\tau_{1}$ and $\tau_{4}$. The existence of two peaks is confirmed by the AmII band around His16-Val17 during period $\tau_{3}$ in Figure S2. The AmII band around His16-Val17 during periods $\tau_{2}$ and $\tau_{4}$ shifted to a lower frequency as shown in Figure 5 but in Figure S2, which represents frequencies of C $-\mathrm{N}$ stretching, this band is only wider around the similar frequency of periods $\tau_{1}$ and $\tau_{3}$.

AmI bands around the hinge during periods $\tau_{2}$ and $\tau_{3}$ had fewer changes compared to AmIII and AmII bands. As shown in Figure S3, AmI bands around Gly14-Lys15 and His16Val17 shifted slightly to higher frequencies during periods $\tau_{2}$ and $\tau_{3}$. This contrasts with the absence of a shift around Ala10-Gly14 for $\tau_{4}$. Due to their larger changes, we can state that AmII and especially AmIII are more sensitive amide bands compared to AmI for this conformational change of making a hinge in LM7-2.

Although we can recognize local differences in shapes and frequencies of individual amide bands between the hinge and helical conformations in molecular simulations, it could be challenging to observe these differences in the Raman and IR spectra of the whole peptide. Amide bands in experimental spectra combine contributions from all amide bands in the peptide. ${ }^{36,41,44}$ If we sum all spectra in Figures 5, S2, and S3, the result will be similar to a combination of Raman and IR spectra. However, experimental spectra include other possible vibrational modes that could overlap with these amide bands ${ }^{36,41,44}$ and lack peaks that are forbidden by selection rules. Real spectra also weight the helix and helix-hinge-helix contributions by their relative probabilities of occurring within the many peptide molecules that are analyzed simultaneously. 


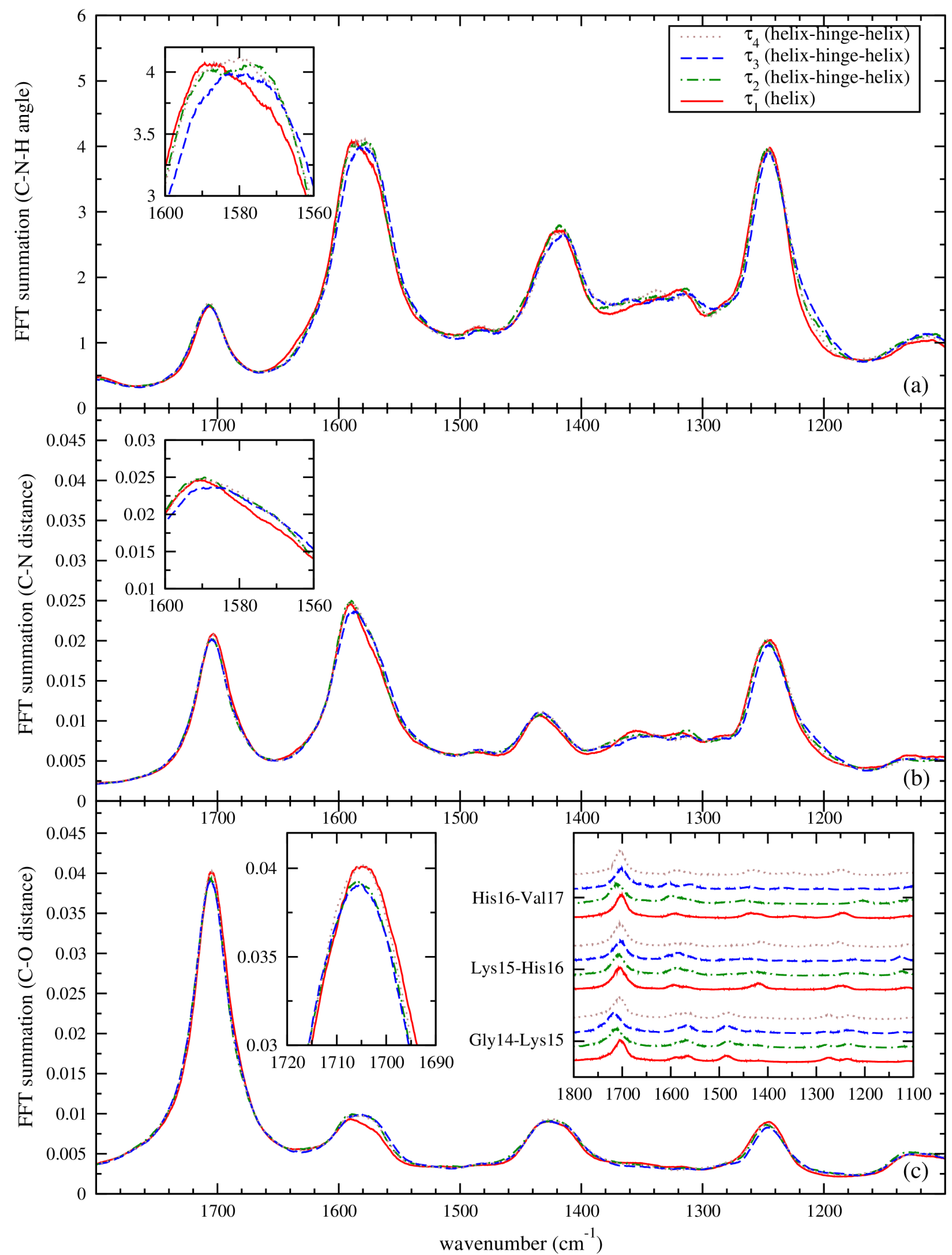

Figure 6: The summation of (a) $\mathrm{C}-\mathrm{N}-\mathrm{H}$ angle bending, (b) $\mathrm{C}-\mathrm{N}$ stretching, and (c) $\mathrm{C}=\mathrm{O}$ stretching FT spectra over all residues for each time period. The AmII peak is magnified in the insets of (a) and (b). The left magnified inset of (c) shows a slight upfield shift of AmI in the presence of a hinge. The right inset of (c), which is available for all amide groups in Figure S3, shows that AmI bands for period $2 \breve{T}_{2}$ and $\tau_{3}$ shifted to higher frequencies around the hinge (Gly14-Lys15). 
The summation of all bands in a single time period are represented by the summation label in Figures 5, S2, and S3 and also are plotted at a higher resolution in Figures 6a-c respectively. The impact on cumulative vibrations varied with the type of internal coordinate. The combined AmIII bands show some slight differences mostly on the peak widths. The peak is slightly more narrow during period $\tau_{1}$, when the whole LM7-2 had a helical conformation, and slightly wider during period $\tau_{3}$, when LM7-2 had the most bent conformation (Figures $6 \mathrm{a}$ and $6 \mathrm{~b}$ ). The width of the combined AmIII band during period $\tau_{2}$, a less bent hinge, increases less than during periods $\tau_{1}$ and $\tau_{3}$. The widths of the combined contributions to AmII bands by $\mathrm{C}-\mathrm{N}-\mathrm{H}$ angles are similar in helix and helix-hinge-helix conformations, as shown in Figure 6a, though there are slight differences especially at the tops of peaks. The top of the $\mathrm{C}-\mathrm{N}-\mathrm{H}$ AmII peak points slightly to a higher frequency $\left(\sim 1587 \mathrm{~cm}^{-1}\right)$ during period $\tau_{1}$ and to a lower frequency $\left(\sim 1577 \mathrm{~cm}^{-1}\right)$ during period $\tau_{3}$. LM7-2 has the most bent conformation during period $\tau_{3}$ and the most helical during period $\tau_{1}$. As shown in all of these figures, and especially Figure $6 \mathrm{c}$, which directly represents $\mathrm{C}=\mathrm{O}$ stretching, the combined AmI bands have a similar shape and width, while the frequency shifted slightly higher for periods $\tau_{2}$ and $\tau_{3}$.

The changes in peak width, shape, and frequency suggest opportunities to identify the presence of multiple conformations in sufficiently sensitive experiments. In a complete helical state (period $\tau_{1}$ ), AmII band had a frequency at $1587 \mathrm{~cm}^{-1}$ and a strong contribution above $1570 \mathrm{~cm}^{-1}$ that appears as a shoulder (see fig 6a inset). In the most bent conformation (period $\tau_{3}$ ), the $1587 \mathrm{~cm}^{-1}$ peak became a shoulder while the peak around $1577 \mathrm{~cm}^{-1}$ dominated. The top of the AmII band during period $\tau_{2}$ split into two frequencies: one frequency is close to the frequency of period $\tau_{1}\left(\sim 1587 \mathrm{~cm}^{-1}\right)$ and another to the frequency of period $\tau_{3}\left(\sim 1577 \mathrm{~cm}^{-1}\right)$. LM7-2 during period $\tau_{2}$ bent less compared to during period $\tau_{3}$. Since the structure of LM7-2 is between the most bent and the most helical conformation, its AmII peak shows a combination of peaks of periods $\tau_{1}$ and $\tau_{3}$. We can state that LM7-2 during period $\tau_{2}$ is a mid-phase conformation in the transition of LM7-2 from complete helical 
conformation to the most bent conformation. AmII band during period $\tau_{4}$ covers both of these split frequencies with a peak close to that of the $\tau_{3}$ period. The trend of changes in the combined AmIII band from the most narrow peak during period $\tau_{1}$ to the widest peak during period $\tau_{3}$ could help to monitor a transition between helical and helix-hinge-helix conformations. The AmI band shifted rather than changed shape in the presence of a hinge (right inset of Figure 6c, Figure S3).

A bending transition of a helical LM7-2 around Lys15-Val17 (from $\tau_{1}$ to $\tau_{2}$ and then to $\tau_{3}$ ) can be monitored by combined AmII and AmIII bands. The exact position of the hinge cannot be determined by combined bands. However, our results in Figures 5, S2, and S3, which show each amide band of each amide group, suggest that separating individual amide group spectra can clarify possible positions of the hinge.

The sensitivities of changes in peak width, shape, and frequency are consistent with prior observations in experiments. While sensitivity of AmI to slight changes of helical peptides has been identified and used in experimental studies, some studies suggest that AmIII can be even more sensitive. Frey and Tamm ${ }^{97}$ applied polarized attenuated total reflection IR to parallel and perpendicular orientations of $\alpha$-helical melittin in a membrane. Spectra showed that the AmI band was similar between these two orientations. Zhang et al. ${ }^{98}$ studied FTIR spectra of an $\alpha$-helical Lys2-Gly-Leu24-Lys2-Ala-amide peptide during the phospholipid phase transition from liquid-crystalline to gel phase. The AmI band shifted slightly from $\sim 1655$ to $\sim 1658 \mathrm{~cm}^{-1}$, which they explained as an increase of intramolecularhelical hydrogen bond lengths, and thus a stretch of the helical peptide, in the gel-phase. Xiong and Asher ${ }^{50}$ applied temperature-jump UVRR from 10 to $\sim 50^{\circ} \mathrm{C}$ to study (un)folding of poly-L-lysine. The $\alpha$-helix melted with increasing temperature and the AmI band shifted to slightly higher frequency, while significant changes were observed around the AmIII band. Ma et al. ${ }^{49}$ applied this method to a similar system at different salt concentrations; they found that the AmIII band was the most sensitive to the conformational changes at different salt concentration. 
Isotope labels have enabled spectroscopists to distinguish contributions from different amide groups. Maekawa et al. brought about distinct AmI shifts and slight AmII shifts in 2D IR for a hydrogen bonded ${ }^{13} \mathrm{C}={ }^{18} \mathrm{O} \cdots \mathrm{H}-{ }^{15} \mathrm{~N}$ pair in a 310 helix. ${ }^{76}$ Woys et al. applied 2D IR on specially formulated AMPs with ${ }^{13} \mathrm{C}={ }^{18} \mathrm{O}$ labeling on one amide group. ${ }^{32} \mathrm{~A}$ comparison of results from all possible single-site labels showed that the inhomogeneous linewidth in 2D IR was sensitive to the local polarity environment. This indicated which groups of the AMP faced the nonpolar tail or the polar head group when it was immersed in a lipid bilayer. These works support the idea that an amide group-specific measurement can yield information about molecular-scale changes, such as hydrogen bonding and local polarity. We suggest that local conformation may alter peak position and shape in a detectable manner.

Investigating the position or even the existence of a hinge for a helical peptide from typical Raman or IR spectra is more difficult. The spectra obtained by Raman or IR are more complicated around AmII and AmIII bands because other kinds of vibrational modes may overlap these amide bands. Another challenge for detecting a hinge by experimental vibrational spectroscopy is its unstable conformation and its appearance for only a short time period, which make a hinge conformation rare among the many molecules whose vibrations are measured simultaneously. For example, Hawrani et al. report that high peptide and lipid concentrations are required to detect AMP conformation effects near a lipid bilayer when using circular dichroism. ${ }^{31}$ Still, such measurements may be possible. Venanzi et al. ${ }^{99}$ considered an AMP that contains an a rare aminoisobutyric acid residue, which sterically altered peptide dynamics. By measuring fluorescence lifetime and computing quench lifetimes for different conformations, they identified forward and reverse rate constants of $1 /(320 \mathrm{~ns})$ and 1/(58 ns) for conversions from an $\alpha$-helix to a helix-turn-helix conformation, which indicates a long-lived turn compared to the hinge lifetimes of under $0.2 \mathrm{~ns}$ found here for LM7-2. Their results suggest that presence of a hinge can be measurable if its lifetime is sufficiently long compared to an experiment. 


\section{Normal Mode Analysis (NMA) Methods}

Ala-Ala (AA) dipeptide was used as a reference molecule to characterize the vibrational movements of amide bands. NMA was applied on the minimized structure of AA dipeptide with a neutral amine N-terminus and neutral carboxyl C-terminus. By watching animations of AA vibrations that were obtained on the basis of eigenvectors, we could specify AmI at $1689.12 \mathrm{~cm}^{-1}$ (Movie AmI), AmII at $1579.81 \mathrm{~cm}^{-1}$ (Movie AmII), and AmIII at $1220.28 \mathrm{~cm}^{-1}$ (Movie AmIII). The eigenvectors of these three modes were considered as reference vectors for monitoring these amide bands in LM7-2 over MD time steps and in other AA dipeptide structures. Moreover, NMA was implemented on a minimized AA dipeptide with protonated amine $\left(\mathrm{NH}_{3}{ }^{+}\right)$and carboxylate $\left(\mathrm{COO}^{-}\right)$terminal groups, a minimized AA dipeptide with methylamide terminal groups, and neutral and zwitterionic AA dipeptides with helical torsion angles. The dot product method was used to identify amide bands among the eigenvectors. Although all amide bands shifted to lower or higher frequencies via various terminal groups and various torsion angles, the movements of atoms in these bands were the same as for the minimized AA dipeptide with neutral end groups. Table S1 provides $\psi, \varphi$, and amide band wavenumbers for these five AA dipeptide structures.

With the LM7-2 system, we first applied NMA at $\sim 40$ ns (Figure 3a) and $\sim 60.7$ ns (Figure 3b) as examples of helical and most bent helix-hinge-helix conformations, respectively. The number of normal modes within each $5 \mathrm{~cm}^{-1}$ was tabulated for NMA results of each conformation. Figure 7 shows this number of normal modes vs. wavenumber for each conformation. There were some negative eigenvalues, which we show at their corresponding wavenumbers $i \nu$ by a negative sign. Since LM7-2 was not in its minimum energy structure over the MD simulation, the existence of negative eigenvalues was expected. This graph is similar to vibrational spectra such as Raman or IR that include all possible vibrational information of the whole peptide.

Investigating this kind of computed complex spectra is always challenging because, similar to experimental spectra, there are several overlapping bands ${ }^{36,41,44}$ such as overlap of 


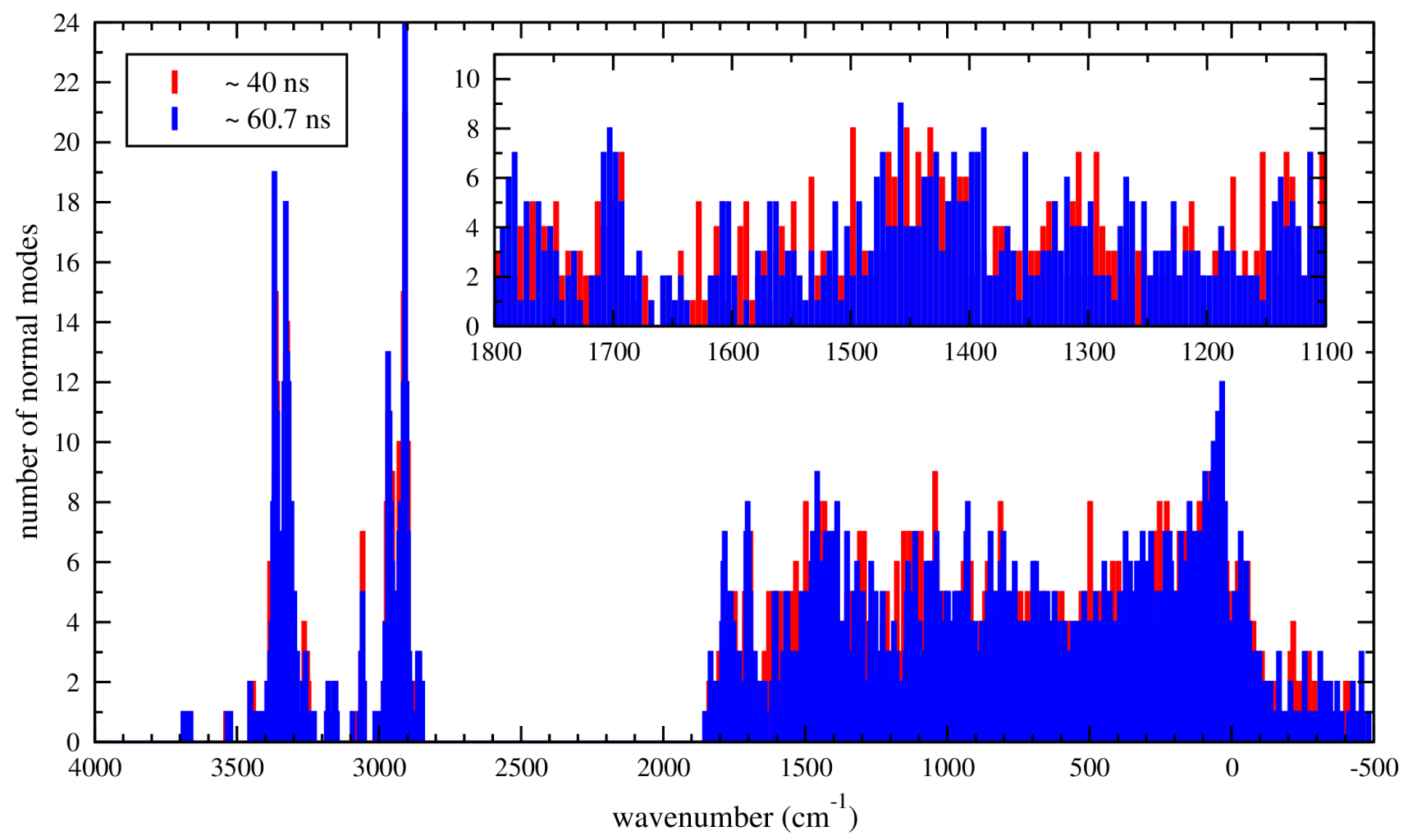

Figure 7: Normal mode spectra of LM7-2 at $\sim 40$ ns and $\sim 60.7$ ns in which LM7-2 has helical and helix-hinge-helix conformations, respectively.

side chain vibrations with AmIII modes. ${ }^{36,44}$ In addition, because each amide band is the summation of coupled modes of all amide groups through the peptide backbone, ${ }^{44}$ and most amide groups retain similar geometries in these conformational states on the basis of Figure 1, interpreting and comparing the spectra of these two states of LM7-2 are even more challenging. Finding differences between the spectra of these two states relies on differences due to the hinge around Lys15-Val17 at $\sim 60.7$ ns.

Identifying amide band contributions from each amide group of LM7-2 by investigating all eigenvectors or watching the animation of each mode is inconvenient. Hence, we applied the dot product technique, explained in the methodology, to find amide modes for each amide group of LM7-2. To clarify this method, we illustrate an example of finding the AmI mode between residues His16 and Val17 at $\sim 40$ ns. First, the reference AA dipeptide was aligned on the amide group between His16 and Val17. On the basis of this alignment, all eigenvectors were oriented. Then, the reference AmI eigenvector was reduced by keeping (x, 
$\mathrm{y}, \mathrm{z}$ ) components of only atoms $\mathrm{C}, \mathrm{N}$, and $\mathrm{O}$ of the AA amide group. All 3N eigenvectors of LM7-2 were reduced by keeping $(\mathrm{x}, \mathrm{y}, \mathrm{z})$ components of only atoms $\mathrm{C}, \mathrm{N}$, and $\mathrm{O}$ of the amide group between His16 and Val17. Since eigenvectors are orthonormal, the magnitude of each unreduced eigenvector is one. Hence, the magnitude of each reduced eigenvector, which is a fraction between zero and one, indicates the participation of atoms $\mathrm{C}, \mathrm{N}$, and $\mathrm{O}$ of the His16-Val17 amide group in that mode. We called this magnitude of a reduced eigenvector the "participant fraction". Then, the reduced reference eigenvector and all LM7-2 reduced eigenvectors were renormalized. Next, the dot products of the reduced reference eigenvector with all $\sim 2000$ eigenvectors of LM7-2 and the neighboring ions and water molecules were calculated. If the dot product was more than 0.9 , we considered that mode as a contributor to AmI for His16-Val17. For instance, our calculations show that 33 modes have a dot product of more than 0.9 yet also unique frequencies and different participant fractions. These results demonstrate that AmI vibrations of His16-Val17 are distributed over some normal modes and frequencies. In animations of these modes, other atoms of LM7-2 vibrate besides the AmI band around His16-Val17. Finally, these calculations were repeated for each amide group of LM7-2. Similar calculations were done to monitor AmII and AmIII through LM7-2 at $\sim 40$ ns. According to these results, amide bands of each amide group were distributed over diverse frequencies and modes. Such involvement of similar atom vibrations in multiple normal modes is consistent with the anharmonic mixing among modes that is observed in 2D IR experiments. ${ }^{32,33,57,58,76}$

To perform sampling that monitors each amide band in solution, instantaneous NMA was applied on the LM7-2 system including neighboring ions and water molecules for all four selected time periods. There are 2000 saved conformations (pdb files) in each time period. NMA was applied on each conformation (pdb file) of each time period. The dot product technique was implemented on each conformation to find AmI, AmII, and AmIII modes of all 26 amide groups of LM7-2. The wavenumbers and participant fractions of a similar mode were stored for each amide mode, each amide group, and each conformation. 
As explained earlier, each amide mode (I, II, or III) of each LM7-2 amide group of each conformation might be distributed over different frequencies and modes. Hence, we calculated an average wavenumber and variance by assigning participant fractions as weights. For each conformation, each amide mode, and each LM7-2 amide group, we calculated the averaged wavenumber and its corresponding variance

$$
\begin{gathered}
\langle\nu\rangle_{i}=\frac{\sum_{j=1}^{n} p_{j} \nu_{j}}{\sum_{j=1}^{n} p_{j}} \\
\sigma_{i}^{2}=\frac{\sum_{j=1}^{n} p_{j}\left(\nu_{j}-\langle\nu\rangle_{i}\right)^{2}}{\sum_{j=1}^{n} p_{j}}
\end{gathered}
$$

where $\langle\nu\rangle_{i}$ and $\sigma_{i}^{2}$ are respectively the averaged wavenumber and its corresponding variance of conformation $i$ that has $n$ similar modes with wavenumbers $\nu_{j} . p_{j}$ is the participant fraction of each mode; note that these do not necessarily sum to 1 over all modes. Then, for each selected time period, we averaged these averaged wavenumbers over 2000 time steps (pdb files), and calculated the overall standard deviation,

$$
\begin{aligned}
& \langle\nu\rangle=\frac{1}{\mathrm{~N}_{\text {conf }}} \sum_{i=1}^{\mathrm{N}_{\text {conf }}}\langle\nu\rangle_{i} \\
& S=\sqrt{\frac{1}{\mathrm{~N}_{\text {conf }}} \sum_{i=1}^{\mathrm{N}_{\text {conf }}} \sigma_{i}^{2}}
\end{aligned}
$$

where $\langle\nu\rangle$ and $S$ are respectively the average and standard deviation of the mode of interest over $\mathrm{N}_{\text {conf }}=2000$ conformations.

The average wavenumbers for all amide bands of all amide groups and for all time periods are shown in Figure 8. Standard deviations are indicated by error bars. The average 
wavenumber and standard deviation can be interpreted as a peak and its width, respectively. We note a very narrow distribution for AmI and broad distribution for AmIII at the chain start for all four periods and at the chain end for a helix. Residues in the terminal groups of LM7-2 moved more freely, and torsion angles $\varphi$ and $\psi$ around terminal residues experienced random coil conformations that were not in the $\alpha$-helix criteria.

To consider helical and helix-hinge-helix geometries, we focused comparisons on residues between Thr5-Thr23. Amide modes for groups within Lys4-Ala11 and Gly18-Leu26 have similar average frequencies and standard deviations among all four time periods because LM7-2 had a helical conformation around these residues throughout the MD simulation.

LM7-2 had a helix-hinge-helix conformation around Ala11-Val13 during period $\tau_{4}$ and a helical conformation around this position during other time periods. As shown in Figure 8, the AmIII mode around Ala11-His12 and His12-Val13 during period $\tau_{4}$ shifted to a lower frequency compared to other time periods. The AmII mode of these residues during period $\tau_{4}$ shifted slightly to a lower frequency and had broader standard deviations around Ala11His12 and His12-Val13. The frequency and the standard deviation of the AmI mode of these residues were similar for all four time periods. These results are in good agreement with FT method results in Figures 5, S2, and S3, where similar shifts for AmIII and AmII were observed. During periods $\tau_{2}$ and $\tau_{3}$, LM7-2 had a helix-hinge-helix conformation around Lys15-Val17. In both time periods, the AmIII mode shifted to a lower frequency around Lys15-His16 and His16-Val17. The shift was more significant around His16-Val17. During both periods $\tau_{2}$ and $\tau_{3}$, the standard deviations of the AmIII mode around Lys15-Val17 and the AmII mode around Lys15-His16 were broader, which shows that AmIII and AmII modes experienced wider frequency ranges. Average AmII modes of periods $\tau_{2}$ and $\tau_{3}$ were shifted to a lower frequency compared to two time periods of helical conformations. Similar shifts were also observed in Figures 5 and S2. Moreover, the AmI band was slightly wider around Gly14-His16 during periods $\tau_{2}$ and $\tau_{3}$ compared to periods $\tau_{1}$ and $\tau_{4}$ (Figure 8 ), and it was tighter during period $\tau_{3}$ around His16-Val17, when LM7-2 had the most bent 


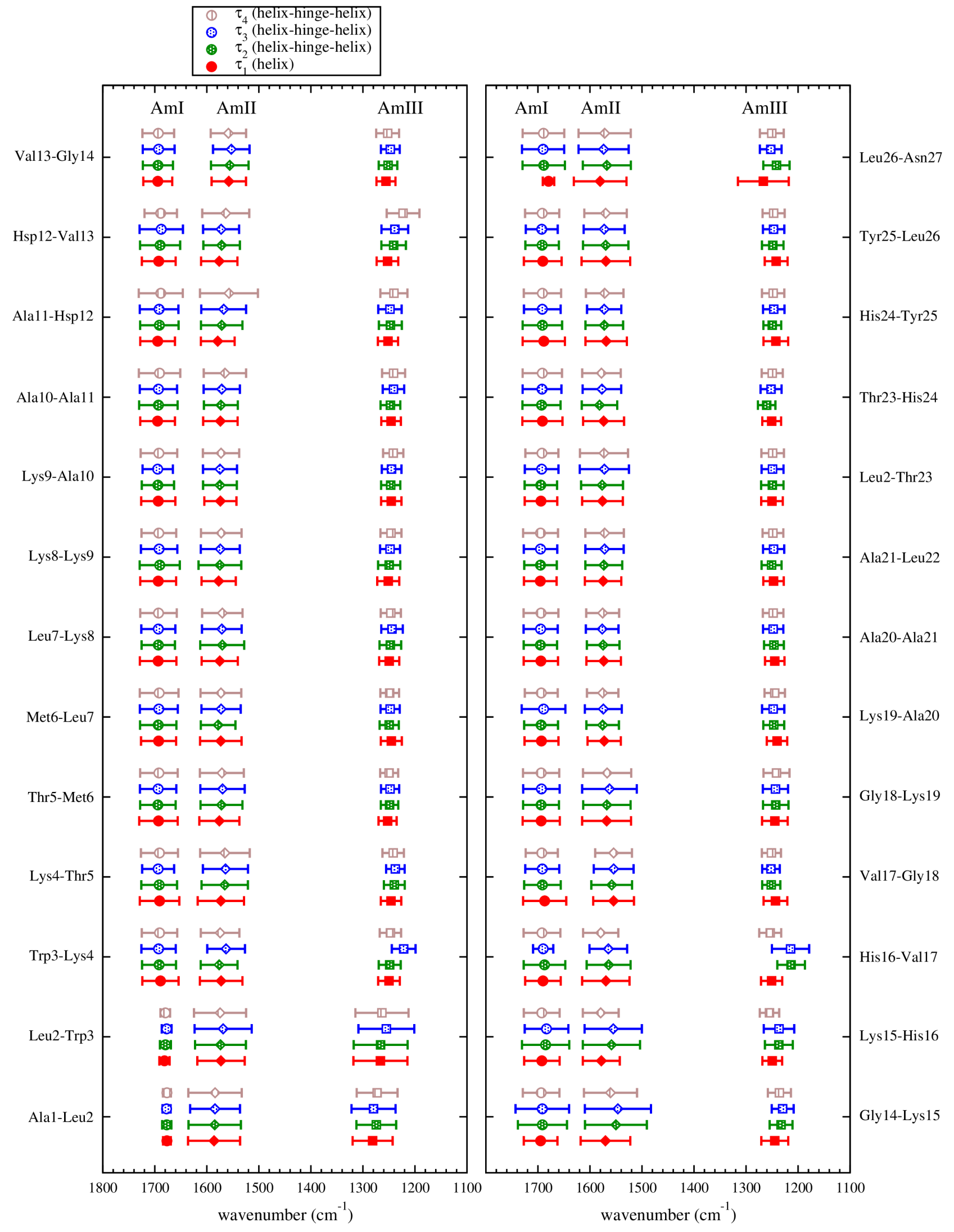

Figure 8: Instantaneous NMA results of each amide band and each position through LM7-2 for all selected four time periods. 
conformation. The instantaneous NMA results confirm the dependency of amide bands especially AmII and AmIII — on the secondary structure of LM7-2. Changes in average and standard deviation of AmII and AmIII modes could help to identify the presence and position of the hinge. These results are in good agreement with the results of the FT method.

To confirm that 2000 conformations are adequate for averaging wavenumbers of each amide mode obtained by instantaneous NMA, we repeated the averaging process over a range of 20 to 1000 conformations. Each new set of conformations was selected by applying different intervals to these same 2000 conformations. For example, 1000 conformations were selected with an interval of 2 among 2000 conformations. Figure S4 demonstrates these averages of AmIII at His17-Val18 for each of four time periods. In all time periods, the averaged wavenumbers and standard deviations were the same for the range of 500-2000 conformations, which validates that 2000 conformations are reliable in terms of averaging.

\section{Hydrogen Bonding}

One crucial effective force in the formation of secondary structure of proteins or peptides and in their folding process is hydrogen bonds. ${ }^{100}$ Peptides or proteins can have intramolecular hydrogen bonds (HBs) mostly between hydrogen (HN) of an amide group and nitrogen (N) or oxygen $(\mathrm{O})$ of another amide group. In addition, they can have intermolecular HBs between $\mathrm{HN}$ and oxygen $(\mathrm{OH} 2)$ of water molecules or $\mathrm{O}$ of amide and hydrogen $(\mathrm{H})$ of water

molecules. ${ }^{101}$ To compare and investigate HBs of LM7-2 with helical and helix-hinge-helix conformations, the radial distribution function $g(r)$ was calculated with $\mathrm{VMD}^{80}$ software between $\mathrm{HN}$ and $\mathrm{OH} 2, \mathrm{HN}$ and $\mathrm{O}$, and $\mathrm{O}$ and $\mathrm{H}$ during four selected time periods.

Figure 9 shows the results of these calculations for two amide groups: Leu7-Lys8 as a region that remained in a helical conformation during 100 ns MD simulation, and His16Val17 that experienced a helix-hinge-helix conformation during short time periods such as $\tau_{2}$ and $\tau_{3}$. As implied in Figure 9a, $g(r)$ between atom HN of the amide group in Leu7-Lys8 and $\mathrm{OH} 2$ atoms of water molecules retained a similar shape during the four different time 
periods. It demonstrates that the concentration of water molecules closer than $\sim 10 \AA$ to atom HN at Leu7-Lys8 is less than average during MD simulation while LM7-2 remained in a helical conformation. The water concentration is essentially zero closer than $\sim 3-4 \AA$. However, as shown in Figure 9b, differences were observed for equivalent $g(r)$ in His16-Val17 during the four time periods. In the presence of a hinge, there are separations between $\mathrm{HN}$ and $\mathrm{OH} 2$ atoms at $2.25 \AA$ during period $\tau_{3}$ and at $4.0 \AA$ during periods $\tau_{2}$ and $\tau_{3}$ that are in the possible hydrogen bond range. These peaks did not exist in periods $\tau_{1}$ and $\tau_{4}$, when His16-Val17 was a helix. The presence of a hinge during periods $\tau_{2}$ and $\tau_{3}$ enables intermolecular hydrogen bonding of $\mathrm{OH} 2$ of water molecules with $\mathrm{HN}$ of the amide group in His16-Val17.

For intramolecular peptide associations, $g(r)$ between atom HN of the amide group in Leu7-Lys8 and atom O of other LM7-2 amide groups, shown in Figure 9c, demonstrates only small differences among all four time periods, which indicates a similar distribution of $\mathrm{O}$ atoms with respect to atom $\mathrm{HN}$ in Leu7-Lys8. In comparison, for the same $g(r)$ calculation around His16-Val17 (Figure 9d), sharp peaks around $2 \AA$ were observed only during periods $\tau_{1}$ and $\tau_{4}$ when LM7-2 had a helical conformation. Only then did HN between these residues have access to $\mathrm{O}$ atoms of neighboring amide groups in the range of possible hydrogen bonding. This chance was almost zero when LM7-2 had a hinge around atom HN. The extreme magnitude of $g(r)$, with peaks above 500 compared to the average density at $g(r)=$ 1 , indicate the precision of these hydrogen bond locations within a helical conformation.

Changes in $g(r)$ when a helix transforms to a helix-hinge-helix are found in other amide groups as well. Figures S5 and S6 present $g(r)$ between atoms $\mathrm{HN}$ and $\mathrm{OH} 2$ and $g(r)$ between atoms HN and O, respectively, for all amide groups in LM7-2. On the basis of Figures S5, intermolecular HN-OH2 peaks at $r$ of less than $4 \AA$ were observed around residues from His16 to Lys19 only during periods $\tau_{2}$ and $\tau_{3}$ when LM7-2 had a hinge around Lys15-Val7. For His16 to Lys19, $g(r)$ between HN and O in Figure S6 shows sharp intramolecular peaks around $2 \AA$ during periods $\tau_{1}$ and $\tau_{4}$ when LM7-2 has a helical conformation. However, 

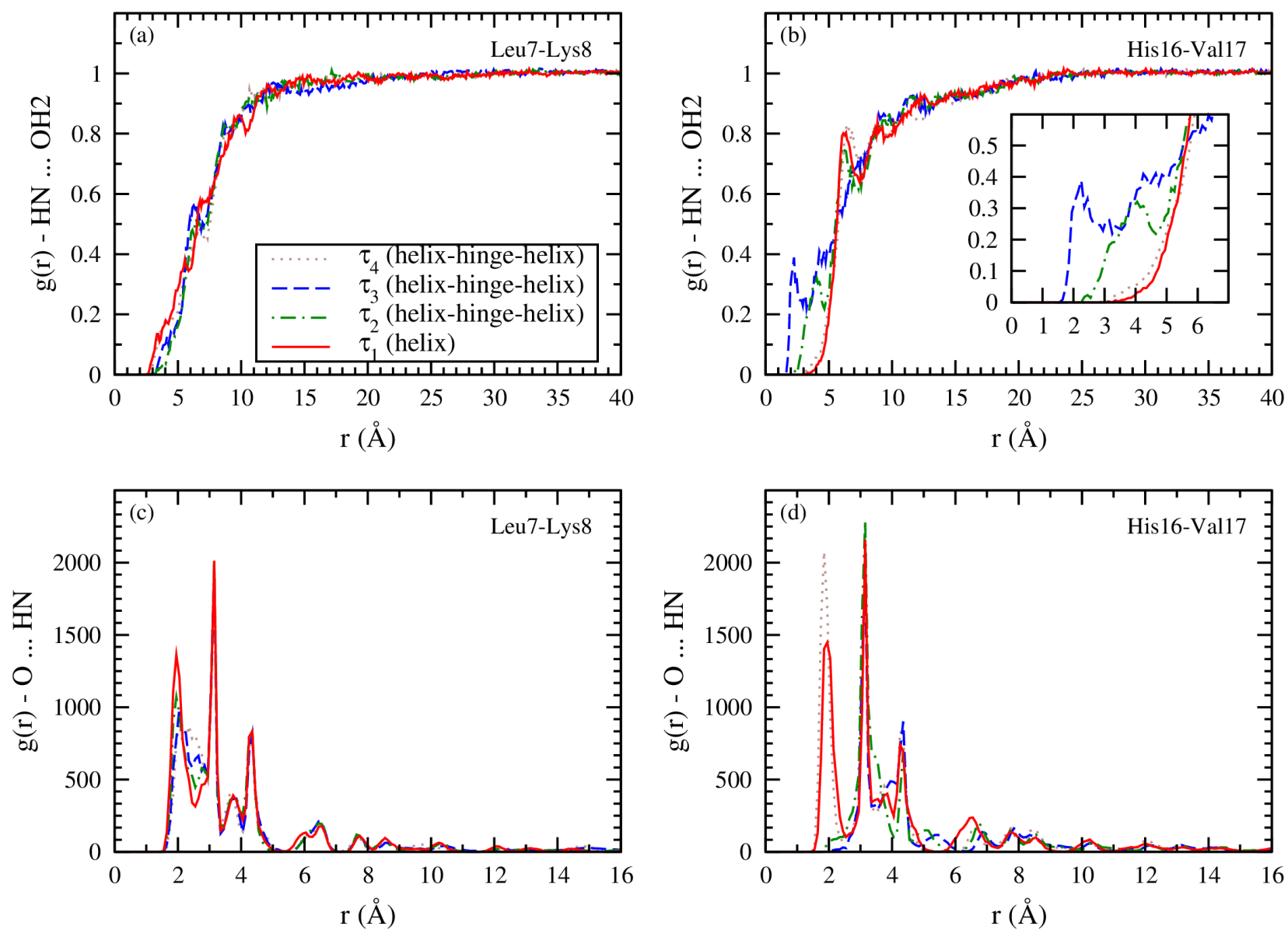

Figure 9: Radial distribution functions that compare $(\mathrm{a}, \mathrm{b})$ intermolecular peptide-water and $(\mathrm{c}, \mathrm{d})$ intramolecular peptide-peptide hydrogen bonds for amide groups that $(\mathrm{a}, \mathrm{c})$ remain helical or $(\mathrm{b}, \mathrm{d})$ experience a transition to a helix-hinge-helix.

these peaks do not exist during periods $\tau_{2}$ and $\tau_{3}$ when LM7-2 has a hinge around Lys16Val17. It confirms that $\mathrm{HN}$ atoms of these residues do not have access to $\mathrm{O}$ atoms of other amide groups in the range of hydrogen bonding during periods $\tau_{2}$ and $\tau_{3}$. During period $\tau_{4}$ when LM7-2 has a hinge around Ala10-Val13, $g(r)$ peaks between atoms HN and OH2 with $r$ of less than $4 \AA$ were observed around His12-Gly14 (Figure S5). These peaks were not observed for other time periods, when LM7-2 remained in a helical conformation. Around His12-Gly14, $g(r)$ between atoms HN and O (Figure S6) show peaks around $2 \AA$ for all time periods except $\tau_{4}$; however, peaks are sharper during time period $\tau_{1}$, especially around Val13-Gly14 compared to time periods $\tau_{2}$ and $\tau_{3}$ when these residues are located close to the hinge. It indicates that close access of atom HN in His12-Gly14 residues to 
neighboring $\mathrm{O}$ atoms is almost zero during $\tau_{4}$. These results confirm that when LM7-2 has a helix-hinge-helix conformation, atoms $\mathrm{HN}$ of amide groups at residues around the hinge are more likely to make intermolecular hydrogen bonds with water molecules rather than intramolecular hydrogen bonds with $\mathrm{O}$ atoms of neighboring amide groups. HN atoms of these residues tended to make intramolecular rather than intermolecular hydrogen bonds when LM7-2 experiences a helical conformation.

The local changes in $\mathrm{N}-\mathrm{H} \cdots \mathrm{O}$ hydrogen bonding between intramolecular (helix) and intermolecular (hinge) neighbors are consistent with shifts in the frequency results. In $2 \mathrm{D}$ IR experiments on a short isotope-substituted peptide, an AmII red shift of $\sim 30-50 \mathrm{~cm}^{-1}$ was found when intramolecular hydrogen bonding decreased. ${ }^{76}$ In spectra from both FT and instantaneous NMA, we found a similar red shift for the hinge compared to the helix. For the most bent case, the AmII peak decreased in FT from 1590 (period $\tau_{1}$ ) to $1560 \mathrm{~cm}^{-1}$ (period $\tau_{3}$ ) around His16-Val17 in Figures 5 and S2. In NMA, the decrease was from 1570 to $1546 \mathrm{~cm}^{-1}$ around Gly14-Lys15 and 1578 to $1555 \mathrm{~cm}^{-1}$ around Lys15-His16 in Figure 8. In the presence of a hinge, and the corresponding loss of intramolecular hydrogen bonding, the AmI peak shown by $\mathrm{C}=\mathrm{O}$ bond fluctuations shifts to higher frequency under the same conditions that the AmII peak shown by $\mathrm{C}-\mathrm{N}$ bond and $\mathrm{C}-\mathrm{N}-\mathrm{H}$ angle fluctuations shifts to lower frequencies.

Figure S7 presents calculated $g(r)$ between $\mathrm{O}$ of each amide group and $\mathrm{H}$ of water molecules for each amide group through LM7-2 during all selected time periods. On the basis of these $g(r)$ results, this pairwise interaction does not show consistent correlation with the existence of the hinge. For example, there is not any hinge around Ala20-Ala21 for these time periods; however, a peak at $r$ of less than $4 \AA$ was observed for all time periods except period $\tau_{2}$, in which LM7-2 has a similar conformation to period $\tau_{3} . g(r)$ around Gly14-Lys15 are similar at $r$ of less than $4 \AA$ for all time periods, yet there is not any hinge around these residues during time periods $\tau_{1}$ and $\tau_{4}$. In some cases we can see correlation to some extent. For instance, LM7-2 has a hinge around Lys15-His16 during periods $\tau_{2}$ and $\tau_{3}$, and 
$g(r)$ showed a sharper peak at $r$ of less than $4 \AA$ around Lys15-His16 during these time periods compared to other periods $\tau_{1}$ and $\tau_{4}$. These observations indicate that hydrogen bonds between $\mathrm{O}$ atoms of all amide groups through the peptide and atom $\mathrm{H}$ of water molecules were not dependent only on the helical or helix-hinge-helix conformation. At most of the positions through the peptide, hydrogen bonds between atoms $\mathrm{O}$ and $\mathrm{H}$ were possible, as shown by peaks around $1.8 \AA$ during all time periods. However, the intensity of these peaks were different for each time period and each amide group.

As shown in Figure S5, significant sharp peaks around residues Ala1 to Lys4 and Lys26Asn27 demonstrate high access of $\mathrm{HN}$ atoms in terminal groups to $\mathrm{OH} 2$ atoms of water molecules. These sharp peaks also were observed in Figures S7 for Ala1-Leu2 residue and residues Thr23 to Asn27, which show high access of $\mathrm{O}$ atoms of amide groups in LM72 terminal groups to $\mathrm{H}$ atoms of water molecules. As shown in Figure S6, the access of HN atom of amine group at the beginning of LM7-2 to O atoms of other amide groups is low during all time periods. However, this access is different for other residues in LM72 during different time periods. Since both terminal groups experienced different random conformations, they have access to water molecules to make intermolecular hydrogen bonds rather than intramolecular hydrogen bonds with other amide groups in LM7-2.

In summary, hydrogen bonding trends for amide-water within the peptide show relations to the presence or absence of a hinge. Around the hinge, intermolecular hydrogen bonds between atom HN and oxygen of water molecules are more probable, and intramolecular hydrogen bonds between atom $\mathrm{HN}$ and atom $\mathrm{O}$ of other amide groups are less probable compared to within a local helical conformation. No relationship was found for amide groups near the start or end of the peptide. These findings suggest a mechanism for hinge formation wherein hydrogen bonds to oxygen atoms of water replace intramolecular hydrogen bonds as the peptide backbone folds. 


\section{Conclusions}

Molecular dynamics (MD) simulations were conducted on a helical designed hybrid antimicrobial peptide (AMP) LM7-2 in solution. A geometric analysis of torsion angles $\psi$ and $\varphi$ pointed out that the $\alpha$-helix LM7-2 bent at its middle mostly around residues Lys15-Val17 and also around residues Ala11-Val13 for a short period of time and then returned to its helical conformation. A helix-hinge-helix conformation of LM7-2 is unstable because LM7-2 would stay at this state for only a short period of time. End-to-end distance and radius of gyration results confirmed the existence of a rare hinge conformation for LM7-2 and indicated that LM7-2 had its most bent conformation during a 100 ns MD simulation around Lys15-Val17 residues.

To investigate how a small change in peptide conformation will affect the fluctuations of its atoms, we studied computationally the inherent vibrations of LM7-2 in both an $\alpha$ helix and in three helix-hinge-helix conformations. We applied two vibrational computation methods, Fourier Transform (FT) and instantaneous all-atom normal mode analysis (NMA), on selected time periods that arose in a $100 \mathrm{~ns}$ molecular dynamics simulation using a classical force field (CHARMM36). The base case was one of many periods when LM7-2 formed an $\alpha$-helix $\left(\tau_{1}\right)$. Comparisons were made with infrequent cases of a helix-hinge-helix around Lys15-Val16 $\left(\tau_{2}\right.$ and $\left.\tau_{3}\right)$ and a helix-hinge-helix around Ala11-Val13 $\left(\tau_{4}\right)$. LM7-2 had the most bent conformation during period $\tau_{3}$. For consistency, each time period was 200 ps. Since

amide bands are sensitive to the secondary structure of peptides, ${ }^{30,36,41-47,52,53}$ we focused in this study on AmI, AmII, and AmIII.

Distances $\mathrm{C}=\mathrm{O}$ and $\mathrm{C}-\mathrm{N}$ and angle $\mathrm{C}-\mathrm{N}-\mathrm{H}$ of each amide group in LM7-2 were calculated for each fs of the MD simulation. FT was applied on these results to calculate local $\mathrm{C}=\mathrm{O}$ stretching, $\mathrm{C}-\mathrm{N}$ stretching, and angle $\mathrm{C}-\mathrm{N}-\mathrm{H}$ bending vibrational frequencies of each amide group in LM7-2. Comparing the FT spectra of each time period indicated that the peak shapes and frequencies of AmII and especially AmIII varied around the hinge. AmII and AmIII shifted to a lower frequency for an amide group at the position of a hinge: at His12- 
Val13 when LM7-2 had a hinge around Ala11-Val13 residues $\left(\tau_{4}\right)$ and at His16-Val17 when LM7-2 had a hinge around residues Lys15-Val17 $\left(\tau_{2}\right.$ and $\left.\tau_{3}\right)$. The shape of AmIII changed around residues Lys15-His16, and also the shape of AmII changed at positions Lys15-His16 and His16-Val17 during periods $\tau_{2}$ and $\tau_{3}$ compared to other time periods. AmI showed less sensitivity to the conformational change of LM7-2.

Our FT results provide local vibrational amide bands in high resolution and detail; however, amide bands of an experimental vibrational spectrum of a peptide are summations of all amide bands through the peptide. Hence, we compared the combination of each amide band through LM7-2 during each time period. The combined spectra demonstrated very slight differences around AmII and AmIII bands such that it may be possible to detect the presence of a hinge with infrared or Raman spectroscopy. The width of combined AmIII bands were slightly different between helix and helix-hinge-helix time periods. AmII bands occurred at a similar frequency but differences were found at the top of combined peaks. The combined spectra provide more pure amide bands compared to experimental vibrational spectra because amide bands could overlap with other vibrational modes.

NMA was applied on an $\alpha$-helix and on the most bent LM7-2 in solution. Since we were interested in amide bands, we used a dot product method to focus on their changes over time during molecular dynamics simulations. NMA was applied on a minimized isolated Ala-Ala dipeptide to specify three reference amide modes. These reference modes were used for monitoring and detecting amide bands. Instantaneous NMA was applied on the LM72 system during all four time periods to monitor each amide band and to average each frequency over each time period. The instantaneous NMA results were in agreement with FT results. AmII and AmIII bands showed the most sensitivity to conformational changes of LM7-2. AmII and AmIII bands shifted to lower frequencies around the hinge, and standard deviations of each frequencies for these two bands were wider for the local hinge conformation compared to the local helical conformation.

Radial distribution functions $g(r)$ were calculated between atom $\mathrm{HN}$ of amide groups and 
oxygen of water molecules, atom $\mathrm{HN}$ and $\mathrm{O}$ of amide groups, and atom $\mathrm{O}$ of amide groups and hydrogen of water molecules. The results indicated that the probability of intermolecular hydrogen bonds between atom $\mathrm{HN}$ and oxygen of water molecules is higher in the local hinge conformation compared to the local helical conformation, while the probability of intramolecular hydrogen bonds between atom $\mathrm{HN}$ and atom $\mathrm{O}$ of other amide groups is lower. Although hydrogen bonding of atom $\mathrm{O}$ of amide groups and hydrogen of water molecules differed for each amide group and during all time periods, there were not relations between this type of hydrogen bonding and the presence of a hinge. The hydrogen bonding results are consistent with a hinge formation mechanism in which HN hydrogen bonds to amide oxygen atoms are replaced with hydrogen bonds to oxygen atoms of water.

In this vibrational study, we provided high resolution vibrational spectra and focused on separate contributions to vibrational spectra that arise from distinct changes in amide group conformation - the presence of a hinge - within the same molecule in classical molecular dynamics simulations. We extracted specific amide modes computationally from the overall spectrum of vibrations and studied the effect of conformational change on the mode of inter-

est. Identifying modes at this level of resolution, especially for small changes in the peptide conformation, is challenging for current experimental tools because there are several overlaps among diverse vibrational modes. We hope that this vibrational study can contribute to interpreting the complex vibrational spectra measured for similar peptide systems.

\section{Acknowledgement}

We thank University of Rhode Island Information Technology Services Research Computing for computational resources used in this study.

\section{Supporting Information Available}

The following files are available free of charge. 
- Supporting Information: Table S1 provides amide band frequencies and backbone torsion angles of five different AA dipepetide structures. Figure S1 presents a flowchart of how instantaneous NMA was applied. Figures S2 and S3 show FT results of C-N and $\mathrm{C}=\mathrm{O}$ distances of all amide groups through LM7-2, respectively. Figure S4 provides frequency averages for the AmIII mode at His16-Val17 position for different numbers of MD steps. Figures S5, S6, and S7 provide radial distribution functions between hydrogen $(\mathrm{HN})$ connected to the nitrogen of each amide group and oxygen of water molecules, HN of each amide group and oxygen of amide groups, and oxygen of each amide group and hydrogen of water molecules, respectively.

- Movies AmI.mpg, AmII.mpg, and AmIII.mpg show animations of the amide bands. Movie Hinge.mpeg shows an animation of LM7-2 hinge formation.

\section{References}

(1) Bechinger, B. The structure, dynamics and orientation of antimicrobial peptides in membranes by multidimensional solid-state NMR spectroscopy. Biochim. Biophys. Acta 1999, 1462, 157-183.

(2) Brogden, K. A. Antimicrobial peptides: pore formers or metabolic inhibitors in bacteria? Nat. Rev. Microbiol. 2005, 3, 238-250.

(3) Sato, H.; Feix, J. B. Peptide-membrane interactions and mechanisms of membrane destruction by amphipathic $\alpha$-helical antimicrobial peptides. Biochim. Biophys. Acta 2006, 1758, 1245-1256.

(4) Wang, J.; Dou, X.; Song, J.; Lyu, Y.; Zhu, X.; Xu, L.; Li, W.; Shan, A. Antimicrobial peptides: Promising alternatives in the post feeding antibiotic era. Med. Res. Rev. 2019, 39, 831-859. 
(5) Dathe, M.; Wieprecht, T. Structural features of helical antimicrobial peptides: Their potential to modulate activity on model membranes and biological cells. Biochim. Biophys. Acta 1999, 1462, 71-87.

(6) Marr, A. K.; Gooderham, W. J.; Hancock, R. E. Antibacterial peptides for therapeutic use: obstacles and realistic outlook. Curr. Opin. Pharmacol. 2006, 6, 468-472.

(7) Wu, M.; Maier, E.; Benz, R.; Hancock, R. E. W. Mechanism of interaction of different classes of cationic antimicrobial peptides with planar bilayers and with the cytoplasmic membrane of Escherichia coli. Biochemistry 1999, 38, 7235-7242.

(8) Epand, R. M.; Vogel, H. J. Diversity of antimicrobial peptides and their mechanisms of action. Biochim. Biophys. Acta 1999, 1462, 11-28.

(9) Bocchinfuso, G.; Bobone, S.; Mazzuca, C.; Palleschi, A.; Stella, L. Fluorescence spectroscopy and molecular dynamics simulations in studies on the mechanism of membrane destabilization by antimicrobial peptides. Cell. Mol. Life. Sci. 2011, 68, 22812301.

(10) Torres, M. D.; Sothiselvam, S.; Lu, T. K.; de la Fuente-Nunez, C. Peptide Design Principles for Antimicrobial Applications. J. Mol. Biol. 2019, 431, 3547-3567.

(11) Powers, J.-P. S.; Hancock, R. E. W. The relationship between peptide structure and antibacterial activity. Peptides 2003, 24, 1681-1691.

(12) Wang, K. F.; Nagarajan, R.; Camesano, T. A. Differentiating antimicrobial peptides interacting with lipid bilayer: Molecular signatures derived from quartz crystal microbalance with dissipation monitoring. Biophys. Chem. 2015, 196, 53-67.

(13) Nguyen, L. T.; Haney, E. F.; Vogel, H. J. The expanding scope of antimicrobial peptide structures and their modes of action. Trends Biotechnol. 2011, 29, 464-472. 
(14) Hwang, P. M.; Vogel, H. J. Structure-function relationships of antimicrobial peptides. Biochem. Cell Biol. 1998, 76, 235-246.

(15) Dubovskii, P. V.; Volynsky, P. E.; Polyansky, A. A.; Chupin, V. V.; Efremov, R. G.; Arseniev, A. S. Spatial structure and activity mechanism of a novel spider antimicrobial peptide. Biochemistry 2006, 45, 10759-10767.

(16) Georgescu, J.; Munhoz, V. H. O.; Bechinger, B. NMR structures of the histidine-rich peptide LAH4 in micellar environments: membrane insertion, pH-dependent mode of antimicrobial action, and DNA transfection. Biophys. J. 2010, 99, 2507-2515.

(17) Pukala, T. L.; Brinkworth, C. S.; Carver, J. A.; Bowie, J. H. Investigating the importance of the flexible hinge in caerin 1.1: solution structures and activity of two synthetically modified caerin peptides. Biochemistry 2004, 43, 937-944.

(18) Pukala, T. L.; Boland, M. P.; Gehman, J. D.; Kuhn-Nentwig, L.; Separovic, F.; Bowie, J. H. Solution structure and interaction of Cupiennin 1a, a spider venom peptide, with phospholipid bilayers. Biochemistry 2007, 46, 3576-3585.

(19) Hsiao, Y. W.; Hedström, M.; Losasso, V.; Metz, S.; Crain, J.; Winn, M. Cooperative modes of action of antimicrobial peptides characterized with atomistic simulations: A study on cecropin B. J. Phys. Chem. B 2018, 122, 5908-5921.

(20) Oh, D.; Shin, S. Y.; Lee, S.; Kang, J. H.; Kim, S. D.; Ryu, P. D.; Hahm, K. S.; Kim, Y. Role of the hinge region and the tryptophan residue in the synthetic antimicrobial peptides, cecropin A(1-8)-magainin 2(1-12) and its analogues, on their antibiotic activities and structures. Biochemistry 2000, 39, 11855-11864.

(21) Hutchinson, E. G.; Thornton, J. M. A revised set of potentials for $\beta$-turn formation in proteins. Protein Sci. 1994, 3, 2207-2216. 
(22) Orioni, B.; Bocchinfuso, G.; Kim, J. Y.; Palleschi, A.; Grande, G.; Bobone, S.; Park, Y.; Kim, J. I.; Hahm, K.-s.; Stella, L. Membrane perturbation by the antimicrobial peptide PMAP-23: A fluorescence and molecular dynamics study. Biochim. Biophys. Acta 2009, 1788, 1523-1533.

(23) Lee, J. K.; Gopal, R.; Park, S.-C.; Ko, H. S.; Kim, Y.; Hahm, K. S.; Park, Y. A proline-hinge alters the characteristics of the amphipathic $\alpha$-helical AMPs. PLoS One 2013, 8, 67597 .

(24) Galanth, C.; Abbassi, F.; Lequin, O.; Ayala-Sanmartin, J.; Ladram, A.; Nicolas, P.; Amiche, M. Mechanism of antibacterial action of dermaseptin B2: Interplay between helix-hinge-helix structure and membrane curvature strain. Biochemistry 2009, 48, 313-327.

(25) Sun, D.; Forsman, J.; Woodward, C. E. Molecular simulations of melittin-induced membrane pores. J. Phys. Chem. B 2017, 121, 10209-10214.

(26) Wang, J.; Ma, K.; Ruan, M.; Wang, Y.; Li, Y.; Fu, Y. V.; Song, Y.; Sun, H.; Wang, J. A novel cecropin B-derived peptide with antibacterial and potential anti-inflammatory properties. PeerJ 2018, 6, e5369.

(27) Mittermaier, A.; Kay, L. E. New tools provide new insights in NMR studies of protein dynamics. Science 2006, 312, 224-228.

(28) Zhuravleva, A.; Korzhnev, D. M. Protein folding by NMR. Prog. Nucl. Magn. Reson. Spectrosc. 2017, 100, 52-77.

(29) Kallenbach, N. R.; Lyu, P.; Zhou, H. In Circ. Dichroism Conform. Anal. Biomolecules; Fasman, G. D., Ed.; Plenum Pres: New York, 1996; pp 201-259.

(30) Tamm, L. K.; Tatulian, S. A. Infrared spectroscopy of proteins and peptides in lipid bilayers. Q. Rev. Biophys. 1997, 30, 365-429. 
(31) Hawrani, A.; Howe, R. A.; Walsh, T. R.; Dempsey, C. E. Origin of low mammalian cell toxicity in a class of highly active antimicrobial amphipathic helical peptides. $J$. Biol. Chem. 2008, 283, 18636-18645.

(32) Woys, A. M.; Lin, Y. S.; Reddy, A. S.; Xiong, W.; De Pablo, J. J.; Skinner, J. L.; Zanni, M. T. 2D IR line shapes probe ovispirin peptide conformation and depth in lipid bilayers. J. Am. Chem. Soc. 2010, 132, 2832-2838.

(33) Ghosh, A.; Ostrander, J. S.; Zanni, M. T. Watching proteins wiggle: mapping structures with two-dimensional infrared spectroscopy. Chem. Rev. 2017, 117, 1072610759.

(34) Lednev, I. K.; Karnoup, A. S.; Sparrow, M. C.; Asher, S. A. $\alpha$-Helix peptide folding and unfolding activation barriers: A nanosecond UV resonance Raman study. J. Am. Chem. Soc. 1999, 121, 8074-8086.

(35) Asher, S. A.; Ianoul, A.; Mix, G.; Boyden, M. N.; Karnoup, A.; Diem, M.; SchweitzerStenner, R. Dihedral $\psi$ angle dependence of the amide III vibration: A uniquely sensitive UV resonance Raman secondary structural probe. J. Am. Chem. Soc. 2001, 123, $11775-11781$.

(36) Maiti, N. C.; Apetri, M. M.; Zagorski, M. G.; Carey, P. R.; Anderson, V. E. Raman spectroscopic characterization of secondary structure in natively unfolded proteins: $\alpha$-synuclein. J. Am. Chem. Soc. 2004, 126, 2399-2408.

(37) Mikhonin, A. V.; Ahmed, Z.; Ianoul, A.; Asher, S. A. Assignments and conformational dependencies of the amide III peptide backbone UV resonance Raman bands. J. Phys. Chem. B 2004, 108, 19020-19028.

(38) Mikhonin, A. V.; Bykov, S. V.; Myshakina, N. S.; Asher, S. A. Peptide secondary structure folding reaction coordinate: Correlation between UV Raman amide III fre- 
quency, $\psi$ Ramachandran angle, and hydrogen bonding. J. Phys. Chem. B 2006, 110, 1928-1943.

(39) Huang, C.-Y.; Balakrishnan, G.; Spiro, T. G. Protein secondary structure from deepUV resonance Raman spectroscopy. J. Raman Spectrosc. 2006, 37, 277-282.

(40) Oladepo, S. A.; Xiong, K.; Hong, Z.; Asher, S. A. Elucidating peptide and protein structure and dynamics: UV resonance Raman spectroscopy. J. Phys. Chem. Lett. 2011, 2, 334-344.

(41) Jakubek, R. S.; Handen, J.; White, S. E.; Asher, S. A.; Lednev, I. K. Ultraviolet resonance Raman spectroscopic markers for protein structure and dynamics. Trends Anal. Chem. 2018, 103, 223-229.

(42) Blanch, E. W.; Hecht, L.; Barron, L. D. Vibrational Raman optical activity of proteins, nucleic acids, and viruses. Methods 2003, 29, 196-209.

(43) Zhu, F.; Isaacs, N. W.; Hecht, L.; Barron, L. D. Raman optical activity: A tool for protein structure analysis. Structure 2005, 13, 1409-1419.

(44) Tuma, R. Raman spectroscopy of proteins: From peptides to large assemblies. $J$. Raman Spectrosc. 2005, 36, 307-319.

(45) Barth, A. Infrared spectroscopy of proteins. Biochim. Biophys. Acta - Bioenerg. 2007, 1767, 1073-1101.

(46) Kinalwa, M. N.; Blanch, E. W.; Doig, A. J. Accurate determination of protein secondary structure content from Raman and Raman optical activity spectra. Anal. Chem. 2010, 82, 6347-6349.

(47) Rygula, A.; Majzner, K.; Marzec, K. M.; Kaczor, A.; Pilarczyk, M.; Baranska, M. Raman spectroscopy of proteins: a review. J. Raman Spectrosc. 2013, 44, 1061-1076. 
(48) Asher, S. A.; Mikhonin, A. V.; Bykov, S. UV Raman demonstrates that $\alpha$-helical polyalanine peptides melt to polyproline II conformations. J. Am. Chem. Soc. 2004, 126, 8433-8440.

(49) Ma, L.; Hong, Z.; Sharma, B.; Asher, S. UV resonance Raman studies of the NaClO4 dependence of poly-L-lysine Conformation and hydrogen exchange kinetics. J. Phys. Chem. B 2012, 116, 1134-1142.

(50) Xiong, K.; Asher, S. A. Impact of ion binding on poly-l-lysine (un)folding energy landscape and kinetics. J. Phys. Chem. B 2012, 116, 7102-7112.

(51) Hong, Z.; Damodaran, K.; Asher, S. A. Sodium dodecyl sulfate monomers induce XAO peptide polyproline II to $\alpha$-helix transition. J. Phys. Chem. B 2014, 118, 10565-10575.

(52) Wen, Z. Q. Raman spectroscopy of protein pharmaceuticals. J. Pharm. Sci. 2007, 96, $2861-2878$.

(53) Balakrishnan, G.; Weeks, C. L.; Ibrahim, M.; Soldatova, A. V.; Spiro, T. G. Protein dynamics from time resolved UV Raman spectroscopy. Curr. Opin. Struct. Biol. 2008, 18, 623-629.

(54) Ianoul, A.; Boyden, M. N.; Asher, S. A. Dependence of the peptide amide III vibration on the $\Phi$ dihedral angle. J. Am. Chem. Soc 2001, 123, 7433-7434.

(55) Cai, S.; Singh, B. R. A distinct utility of the amide III infrared band for secondary structure estimation of aqueous protein solutions using partial least squares methods. Biochemistry 2004, 43, 2541-2549.

(56) Schweitzer-Stenner, R.; Eker, F.; Huang, Q.; Griebenow, K.; Mroz, P. A.; Kozlowski, P. M. Structure analysis of dipeptides in water by exploring and utilizing the structural sensitivity of amide III by polarized visible Raman, FTIR-spectroscopy and DFT based normal coordinate analysis. J. Phys. Chem. B 2002, 106, 4294-4304. 
(57) Maekawa, H.; Toniolo, C.; Moretto, A.; Broxterman, Q. B.; Ge, N. H. Different spectral signatures of octapeptide $33^{-}$and $\alpha$-helices revealed by two-dimensional infrared spectroscopy. J. Phys. Chem. B 2006, 110, 5834-5837.

(58) Maekawa, H.; Toniolo, C.; Broxterman, Q. B.; Ge, N.-H. Two-dimensional infrared spectral signatures of $310^{-}$and $\alpha$-helical peptides. J. Phys. Chem. B 2007, 111, 32223235.

(59) López-Peña, I.; Leigh, B. S.; Schlamadinger, D. E.; Kim, J. E. Insights into protein structure and dynamics by ultraviolet and visible resonance Raman spectroscopy. Biochemistry 2015, 54, 4770-4783.

(60) Schlamadinger, D. E.; Wang, Y.; McCammon, J. A.; Kim, J. E. Spectroscopic and computational study of melittin, cecropin A, and the hybrid peptide CM15. J. Phys. Chem. B 2012, 116, 10600-10608.

(61) Kubelka, J.; Keiderling, T. A. Ab initio calculation of amide carbonyl stretch vibrational frequencies in solution with modified basis sets. 1. N-methyl acetamide. J. Phys. Chem. A 2001, 105, 10922-10928.

(62) Besley, N. A. Ab initio modeling of amide vibrational bands in solution. J. Phys. Chem. A 2004, 108, 10794-10800.

(63) Farag, M. H.; Bastida, A.; Ruiz-López, M. F.; Monard, G.; Ingrosso, F. Vibrational energy relaxation of the amide I mode of $\mathrm{N}$-methylacetamide in $\mathrm{D}_{2} \mathrm{O}$ studied through Born-Oppenheimer molecular dynamics. J. Phys. Chem. B 2014, 118, 6186-6197.

(64) Hahn, S. Effective representation of amide III, II, I, and A modes on local vibrational modes: Analysis of ab initio quantum calculation results. J. Chem. Phys. 2016, 145, 164113. 
(65) Yamamoto, S.; Furukawa, T.; Bouř, P.; Ozaki, Y. Solvated states of poly-L-alanine $\alpha$-helix explored by Raman optical activity. J. Phys. Chem. A 2014, 118, 3655-3662.

(66) Mensch, C.; Barron, L. D.; Johannessen, C. Ramachandran mapping of peptide conformation using a large database of computed Raman and Raman optical activity spectra. Phys. Chem. Chem. Phys. 2016, 18, 31757-31768.

(67) Furuta, M.; Fujisawa, T.; Urago, H.; Eguchi, T.; Shingae, T.; Takahashi, S.; Blanch, E. W.; Unno, M. Raman optical activity of tetra-alanine in the poly(L-proline) II type peptide conformation. Phys. Chem. Chem. Phys. 2017, 19, 2078-2086.

(68) Mensch, C.; Bultinck, P.; Johannessen, C. The effect of protein backbone hydration on the amide vibrations in Raman and Raman optical activity spectra. Phys. Chem. Chem. Phys. 2019, 21, 1988-2005.

(69) Baiz, C. R.; Błasiak, B.; Bredenbeck, J.; Cho, M.; Choi, J. H.; Corcelli, S. A.; Dijkstra, A. G.; Feng, C. J.; Garrett-Roe, S.; Ge, N. H. et al. Vibrational spectroscopic map, vibrational spectroscopy, and intermolecular interaction. Chem. Rev. 2020, 120, $7152-7218$.

(70) Małolepsza, E.; Straub, J. E. Empirical maps for the calculation of amide I vibrational spectra of proteins from classical molecular dynamics simulations. J. Phys. Chem. B 2014, 118, 7848-7855.

(71) Cunha, A. V.; Bondarenko, A. S.; Jansen, T. L. Assessing spectral simulation protocols for the amide I band of proteins. J. Chem. Theory Comput. 2016, 12, 3982-3992.

(72) Stratt, R. M. The instantanteous normal modes of liquids. Acc. Chem. Res. 1995, 28, 201-207.

(73) Bastida, A.; Soler, M. A.; Zúñiga, J.; Requena, A.; Kalstein, A.; Fernández-Alberti, S. Instantaneous normal modes, resonances, and decay channels in the vibrational re- 
laxation of the amide I mode of N-methylacetamide-D in liquid deuterated water. $J$. Chem. Phys. 2010, 132, 134110.

(74) Farag, M. H.; Zúñiga, J.; Requena, A.; Bastida, A. Instantaneous normal mode analysis of the vibrational relaxation of the amide I mode of alanine dipeptide in water. J. Chem. Phys. 2013, 138, 224501.

(75) Joodaki, F.; Martin, L. M.; Greenfield, M. L. Planarity and out-of-plane vibrational modes of tryptophan and tyrosine in biomolecular modeling. Phys. Chem. Chem. Phys. 2019, 21, 23943-23965.

(76) Maekawa, H.; De Poli, M.; Moretto, A.; Toniolo, C.; Ge, N. H. Toward detecting the formation of a single helical turn by $2 \mathrm{D}$ IR cross peaks between the amide-I and -II modes. J. Phys. Chem. B 2009, 113, 11775-11786.

(77) Ryder, D. E. Synthesis and bioassay of novel hybrid antimicrobial peptides (AMPs), designed to increase therapeutic activity and decrease hemolysis. Master thesis, University of Rhode Island, 2012.

(78) Patrzykat, A.; Friedrich, C. L.; Zhang, L.; Mendoza, V.; Hancock, R. E. Sublethal concentrations of pleurocidin-derived antimicrobial peptides inhibit macromolecular synthesis in Escherichia coli. Antimicrob. Agents Chemother. 2002, 46, 605-614.

(79) Chou, P. Y.; Fasman, G. D. Prediction of protein conformation. Biochemistry 1974, $13,222-245$.

(80) Humphrey, W.; Dalke, A.; Schulten, K. VMD: visual molecular dynamics. J. Molec. Graph. 1996, 14, 33-38.

(81) Phillips, J. C.; Braun, R.; Wang, W.; Gumbart, J.; Tajkhorshid, E.; Villa, E.; Chipot, C.; Skeel, R. D.; Kalé, L.; Schulten, K. Scalable molecular dynamics with NAMD. J. Comput. Chem. 2005, 26, 1781-1802. 
(82) Best, R. B.; Zhu, X.; Shim, J.; Lopes, P. E. M.; Mittal, J.; Feig, M.; MacKerell Jr., A. D. Optimization of the additive CHARMM all-atom protein force field targeting improved sampling of the backbone $\phi, \psi$ and side-chain $\chi_{1}$ and $\chi_{2}$ dihedral angles. $J$. Chem. Theory Comput. 2012, 8, 3257-3273.

(83) Huang, J.; MacKerell Jr., A. D. CHARMM36 all-atom additive protein force field: Validation based on comparison to NMR data. J. Comput. Chem. 2013, 34, 21352145 .

(84) Huang, J.; Rauscher, S.; Nawrocki, G.; Ran, T.; Feig, M.; de Groot, B. L.; Grubmüller, H.; MacKerell Jr., A. D. CHARMM36m: An improved force field for folded and intrinsically disordered proteins. Nat. Methods 2016, 14, 71-73.

(85) Lamoureux, G.; Harder, E.; Vorobyov, I. V.; Roux, B.; MacKerell Jr., A. D. A polarizable model of water for molecular dynamics simulations of biomolecules. Chem. Phys. Lett. 2006, 418, 245-249.

(86) Plimpton, S. J.; Pollock, R.; Stevens, M. Particle-mesh Ewald and rRESPA for parallel molecular dynamics simulations. Proc Eighth SIAM Conf. Parallel Process. Sci. Comput. 1997.

(87) Martyna, G. J.; Tobias, D. J.; Klein, M. L. Constant pressure molecular dynamics algorithms. J. Chem. Phys. 1994, 101, 4177-4189.

(88) Feller, S. E.; Zhang, Y.; Pastor, R. W.; Brooks, B. R. Constant pressure molecular dynamics simulation: The Langevin piston method. J. Chem. Phys. 1995, 103, 46134621.

(89) Fiorin, G.; Klein, M. L.; Hénin, J. Using collective variables to drive molecular dynamics simulations. Mol. Phys. 2013, 111, 3345-3362. 
(90) Eaton, J. W.; Bateman, D.; Hauberg, S.; Wehbring, R. GNU Octave version 4.2.2 manual: a high-level interactive language for numerical computations. 2018; https: //www.gnu.org/software/octave/doc/v4.2.1/.

(91) nVIDIA CUDA toolkit documentation v10.2.89. 2019; https://docs.nvidia.com/ cuda/.

(92) Anderson, E.; Bai, Z.; Bishof, C.; Blackford, S.; Demmel, J.; Dongarra, J.; Du Croz, J.; Greenbaum, A.; Hammarling, S.; McKenney, A. et al. LAPACK users's guide, 3rd ed.; Society for Industrial and Applied Mathematics, 1999.

(93) MacKerell Jr., A. D.; Feig, M.; Brooks III, C. L. Improved treatment of the protein backbone in empirical force fields. J. Am. Chem. Soc. 2004, 126, 698-699.

(94) MacKerell Jr., A. D.; Feig, M.; Brooks III, C. L. Extending the treatment of backbone energetics in protein force fields: Limitations of gas-phase quantum mechanics in reproducing protein conformational distributions in molecular dynamics simulations. J. Comput. Chem. 2004, 25, 1400-1415.

(95) Hanwell, M. D.; Curtis, D. E.; Lonie, D. C.; Vandermeersch, T.; Zurek, E.; Hutchison, G. R. Avogadro: an advanced semantic chemical editor, visualization, and analysis platform. J. Cheminform. 2012, 4, 17.

(96) Ulo, L.; Cravatt, B. F.; Von Heijne, N. G. H.; Zorko, M.; Land, T.; Niessen, S. Introduction to Ppetides and Proteins; CRC Press: Boca Raton, FL, 2010.

(97) Frey, S.; Tamm, L. K. Orientation of melittin in phospholipid bilayers. A polarized attenuated total reflection infrared study. Biophys. J. 1991, 60, 922-930.

(98) Zhang, Y. P.; Lewis, R. N.; Hodges, R. S.; McElhaney, R. N. Peptide models of helical hydrophobic transmembrane $\alpha$-helical segment of a membrane protein with phos- 
phatidylcholine bilayers: Differential scanning calorimetric and FTR spectroscopic studies. Biochemistry 1992, 31, 11579-11588.

(99) Venanzi, M.; Gatto, E.; Bocchinfuso, G.; Palleschi, A.; Stella, L.; Formaggio, F.; Toniolo, C. Dynamics of formation of a helix-turn-helix structure in a membraneactive peptide: A time-resolved spectroscopic study. ChemBioChem 2006, 7, 43-45.

(100) Manas, E. S.; Getahun, Z.; Wright, W. W.; Degrado, W. F.; Vanderkooi, J. M. Infrared spectra of amide groups in $\alpha$-helical proteins: Evidence for hydrogen bonding between helices and water. J. Am. Chem. Soc. 2000, 122, 9883-9890.

(101) Desiraju, G. R.; Steiner, T. The Weak Hydrogen Bond In Structural Chemistry and Biology (International Union of Crystallography Monographs on Crystallography); Oxford University Press: Oxford and New York, 1999. 


\section{Graphical TOC Entry}

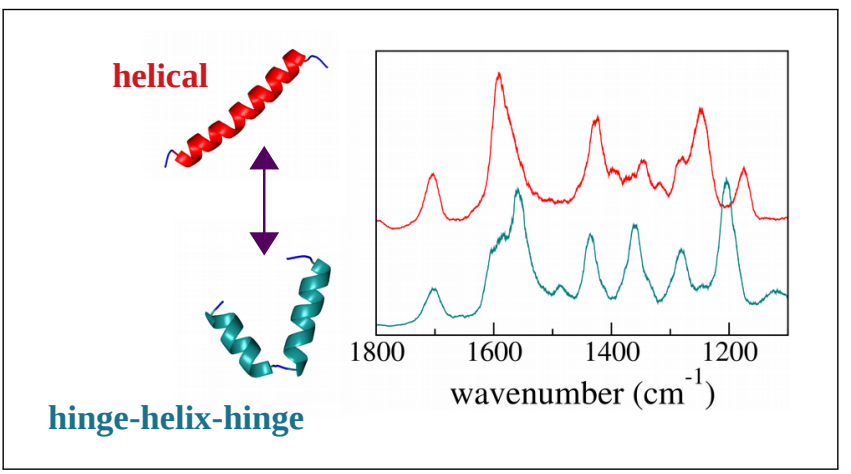

